\title{
A novel dermal matrix generated from burned skin as a promising substitute for deep-degree burns therapy
}

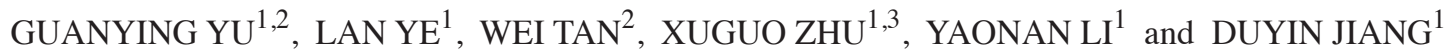 \\ ${ }^{1}$ Department of Burns and Plastic Surgery, The Second Hospital of Shandong University, Jinan, Shandong 250033; \\ ${ }^{2}$ Department of Gastrointestinal Surgery, Jinan Central Hospital, Jinan, Shandong 250013; \\ ${ }^{3}$ Department of Burns and Plastic Surgery, The People's Hospital of Rizhao, Rizhao, Shandong 276800, P.R. China
}

Received May 6, 2015; Accepted January 11, 2016

DOI: $10.3892 / \mathrm{mmr} .2016 .4866$

\begin{abstract}
The extensive skin defects induced by severe burns are dangerous and can be fatal. Currently, the most common therapy is tangential excision to remove the necrotic or denatured areas of skin, followed by skin grafting. Xenogeneic dermal substitutes, such as porcine acellular dermal matrix (ADM), are typically used to cover the burn wounds, and may accelerate wound healing. It is assumed that burned skin that still maintains partial biological activity may be recycled to construct an autologous acellular dermal matrix, termed 'deep-degree burned dermal matrix (DDBDM)'. In theory, DDBDM may avoid the histoincompatibility issues associated with foreign or xenogeneic dermal matrices, and reduce therapy costs by making full use of discarded skin. In the present study, the collagens within prepared DDBDM were thickened, disorganized and partially fractured, however, they still maintained their reticular structure and tensile strength $(\mathrm{P}<0.01)$. Through microarray analysis of the cytokines present in ADM and DDBDM, it was determined that the DDBDM did not produce excessive levels of harmful burn toxins. Following 4 weeks of subcutaneous implantation, ADM and DDBDM were incompletely degraded and maintained good integrity. No significant inflammatory reaction or rejection were observed, which indicated that ADM and DDBDM have good histocompatibility. Therefore, DDBDM may be a useful material for the treatment of deep-degree burns.
\end{abstract}

\section{Introduction}

First-degree burns and superficial wounds undergo a similar wound repair process involving reconstruction of the epithelial barrier, the underlying dermis and extracellular matrix (ECM),

Correspondence to: Professor Duyin Jiang, Department of Burns and Plastic Surgery, The Second Hospital of Shandong University, 247 Beiyuan Street, Jinan, Shandong 250033, P.R. China

E-mail: jdybs2@vip.163.com

Key words: acellular dermal matrix, deep-degree burned dermal matrix, deep burn, cytokine, dermal graft organized by granulation tissue (1). However, second- and third-degree burns present a broad range of additional challenges for restoration of normal dermal function $(2,3)$. The extensive skin defects induced by severe burns are dangerous and can be fatal to patients. A common therapy used to treat extensive burns is skin grafting following tangential excision to remove the necrotic or denatured skin. However, sources of skin transplants are often limited. Xenogeneic dermal substitutes, such as porcine acellular dermal matrix (ADM), can be used to cover the burn wounds, prevent infection, facilitate skin renewal and vascularization, and accelerate wound healing (4-7). However, foreign or xenogeneic tissues have partial histoincompatibility, which can induce rejection and allergic reaction $(8,9)$. Engineered human skin matrices, including Dermagraft, DermACELL and Integra, have exhibited satisfactory effects in the clinic (10-12). However, these skin products are expensive and rare, therefore, their clinical application is restricted. It is important for the clinical therapy of patients with deep second- and third-degree burns to investigate novel dermal substitutes that avoid the limitations associated with currently used dermal matrices.

The necrotic or denatured skin of burn wounds is typically discarded during treatment due to the formation of cutaneous burn toxins (13). Burn toxins, which include free radicals, lipoproteins, lipopolysaccharides and lipid peroxides, are formed as a result of thermal injury to skin and are released into the interstitial fluid and serum during the early stages of burns (14-17). These hazardous substances have been shown to exert various effects, including immunosuppression, mitochondrial destruction, cellular energy metabolic disorders and increasing the permeability of the cell membrane, which in turn have been implicated in the various complications associated with burns, including sepsis and multiple organ dysfunction syndrome (16,18-25). In addition, bacteria can rapidly proliferate in necrotic tissue resulting in wound infection (13). It has been previously observed that sections of the skin removed from deep burn wounds partially maintained the integrity of their collagen structure, and may have the potential to be repaired under the appropriate conditions. Therefore, we hypothesized that burned skin with partial biological activity may be recycled to produce an autologous acellular dermal matrix, termed 'deep-degree burned dermal matrix (DDBDM)', and applied for the treatment of deep burn wounds (26). In theory, 
DDBDM, which originates from human skin, may avoid the histoincompatibility associated with foreign and xenogeneic dermal matrices. Furthermore, DDBDM may reduce therapeutic costs by making full use of the discarded skin. Thus, successful clinical application of DDBDM may be an improved method of treatment for patients with deep burn wounds.

In the current study, DDBDM was successfully prepared using a mouse model of a deep-degree burn wound. To determine if DDBDM and ADM produced burn toxin with different compositions, the protein expression levels of 308 cytokines were analyzed. The DDBDM was subcutaneously implanted in mice to observe whether an inflammatory reaction was induced. The aim of the present study was to investigate the use of DDBDM as a dermal matrix and evaluate its potential clinical significance for the treatment of patients with deep-degree burns.

\section{Materials and methods}

Animals and ethics statement. A total of 150 healthy male Balb/c mice (age, 12 weeks; weight, 27-33 g) were obtained from the Animal Center of Shandong University (Jinan, China). The mice were maintained under a $12 \mathrm{~h}$ light/dark cycle with ad libitum access to animal chow and water in the animal quarter at the Animal Laboratory of the Second Hospital of Shandong University (Jinan, China) at $20-24^{\circ} \mathrm{C}$ and $50-60 \%$ humidity. All experimental procedures were conducted according to the criteria outlined in the Guide for the Care and Use of Laboratory Animals published by the National Institutes of Health (NIH Publication no. 85-23, revised 1996). All experimental protocols were approved by Animal Care and Use Committee of the Second Hospital of Shandong University.

Establishment of burn animal model. A total of 60 healthy male Balb/c mice were used to establish the burn animal model. Following anesthesia by intraperitoneal injection of $10 \%$ chloral hydrate $(0.3 \mathrm{ml} / \mathrm{kg}$; Qilu Hospital, Jinan, China), the fur on the dorsum of each mouse was shaved. The shaved edges were protected by plastic wrap and a thin foam board. A deep-degree burn wound of $5 \times 4 \mathrm{~cm}$ was created by hot water-bath burn, as follows: Using a water bath (Shanghai Jing Hong Experimental Equipment Co. Ltd, Shanghai, China) maintained at a constant temperature of $80^{\circ} \mathrm{C}$, the dorsum of each mouse was bathed in the hot water for $8 \mathrm{sec}$. All the burned mice received anti-shock therapy with Lactated Ringer's solution (Baxter International Inc., Deerfield, IL, USA) by intraperitoneal injection $(40 \mathrm{ml} / \mathrm{kg})$ and were treated with $1 \%$ povidone iodine solution (Lircon Disinfection Science Technology Inc., Dezhou, China) to protect the wound. The mice were resuscitated in a warm environment by subcutaneous injection of physiological saline solution (10 ml/kg; Baxter International, Inc.) until they were fully conscious. After $72 \mathrm{~h}$, the mice were anesthetized and sacrificed by decapitation, after which burned skin was immediately harvested for further experimentation.

ADM preparation method. Following anesthesia and shaving of the fur on the dorsum of 40 healthy Balb/c mice, normal skin specimens were removed and washed with sterile saline solution (Baxter International Inc.). The subcutaneous tissue was removed leaving skin sections of $0.05-1.00 \mathrm{~mm}$ thickness. The skin sections were placed into a mixed solution of $0.25 \%$ trypsin (Sigma-Aldrich, St. Louis, MO, USA) and Triton X-100 (Sigma-Aldrich), and shaken 100 times/min for $2 \mathrm{~h}$ at $37^{\circ} \mathrm{C}$. Samples were repeatedly washed and shaken with phosphate-buffered saline (PBS) until the cells and trypsin/Triton X-100 solution were removed. The ADM was maintained in saline solution with $800 \mathrm{U} / \mathrm{ml}$ gentamicin (Shandong Lukang Chenxin Pharmaceuticals Co., Ltd., Jining, China) at $4^{\circ} \mathrm{C}$. The whole preparation process was conducted under aseptic conditions.

DDBDM preparation method. Burned mouse skin specimens were obtained from 60 burned mice and washed with sterile saline solution. The subcutaneous tissues were removed leaving skin sections of $0.05-1.00 \mathrm{~mm}$ thickness. The skin sections were placed into a mixed solution of $0.25 \%$ trypsin (Sigma-Aldrich) and Triton X-100 (Sigma-Aldrich) and shaken 100 times $/ \mathrm{min}$ for $1 \mathrm{~h}$ at $37^{\circ} \mathrm{C}$. Samples were repeatedly washed and shaken with PBS until the cells and trypsin/Triton X-100 solution were removed. The DDBDM was maintained in saline solution with $800 \mathrm{U} / \mathrm{ml}$ gentamicin at $4^{\circ} \mathrm{C}$. The whole preparation process was conducted under aseptic conditions.

Physical evaluation of $A D M$ and DDBDM. At room temperature and in a humid environment, the prepared ADM and DDBDM were trimmed to $1 \times 1-\mathrm{cm}$ sections and measured with a Benchtop Tester (H10K-T; Tinius Olsen Testing Machine Company, Horsham, PA, USA). The samples were stretched at a rate of $1 \mathrm{~mm} / \mathrm{min}$ until the actual load reached $3 \mathrm{MPa}$, then released at the same rate until the actual load was $0 \mathrm{MPa}$. After repeating the process three times, the stress-strain curve became stable. The strain value at $3 \mathrm{MPa}$ in the fourth stretch was calculated using the following formula: Strain $(\%)=\left[\left(\mathrm{L}-\mathrm{L}_{0}\right) / \mathrm{L}_{0}\right] \times 100=(\mathrm{L}-1) \times 100$, where $\mathrm{L}$ was the length of the dermal matrix during stress in $\mathrm{cm}$ and $\mathrm{L}_{0}$ was the initial length of the dermal matrix, which was $1 \mathrm{~cm}$ in the present study. Subsequently, new samples were stretched at a rate of $1 \mathrm{~mm} / \mathrm{min}$ until broken, recording the values of ultimate tensile strength, maximum tension and elongation at break. All the data were recorded and calculated using a JBK Measure-Control System (Beijing Jincun Electromechanical Technology Research Institute, Beijing, China).

Biotinylated antibody-based cytokine microarray assay. Total protein was extracted from skin samples using RIPA buffer (Beyotime Institute of Biotechnology, Haimen, China), after which the supernatants were obtained by centrifugation at $12,000 \times \mathrm{g}$ for $5 \mathrm{~min}$ at $4^{\circ} \mathrm{C}$. The protein concentrations of the supernatants were detected using a BCA kit (Thermo Fisher Scientific Inc., Waltham, MA, USA), according to the manufacturer's protocol. A total of 308 different mouse proteins, including cytokines, chemokines, adipokines, growth factors, angiogenic factors, proteases, soluble receptors and soluble adhesion molecules, were detected using a mouse cytokine array kit (AAM-BLM-1-4; RayBiotech, Inc., Norcross, GA, USA), according to the manufacturer's protocol. Following subtraction of local background signals, the microarray signals were recorded using a GenePix 4000B microarray scanner (Molecular Devices, LLC, Sunnyvale, CA, USA) using the Cy3 channel and with a scanning 
Table I. Physical properties of acellular and deep-degree burned dermal matrix.

\begin{tabular}{lcccc}
\hline Dermal matrix & $\begin{array}{c}\text { Ultimate tensile } \\
\text { strength, } \mathrm{MPa}\end{array}$ & $\begin{array}{c}\text { Maximum } \\
\text { tension, N }\end{array}$ & $\begin{array}{c}\text { Elongation } \\
\text { at break, } \%\end{array}$ & $\begin{array}{c}\text { Strain at stress of } \\
3 \mathrm{MPa}, \%\end{array}$ \\
\hline $\begin{array}{l}\text { Acellular dermal matrix } \\
\begin{array}{l}\text { Deep-degree burned } \\
\text { dermal matrix }\end{array}\end{array}$ & $15.0 \pm 2.1$ & $84 \pm 4$ & $200 \pm 7$ & $60.1 \pm 2.7$ \\
P-value & $10.2 \pm 1.8$ & $57 \pm 3$ & $182 \pm 5$ & $41.6 \pm 1.4$ \\
\hline
\end{tabular}

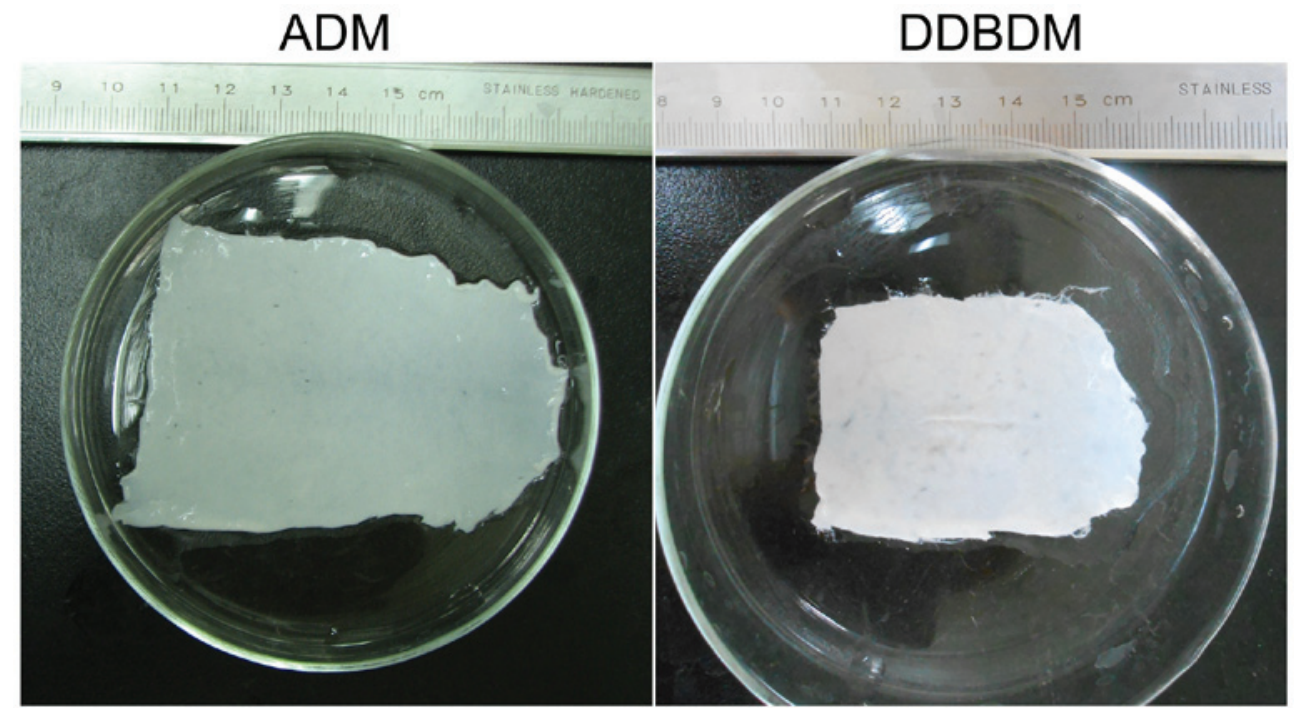

Figure 1. ADM and DDBDM under general observation. ADM, acellular dermal matrix; DDBDM, deep-degree burned dermal matrix.

wavelength of $532 \mathrm{~nm}$. The positive control value was the mean fluorescent signal intensity minus the background of all the positive control spots. Following background subtraction, negative signal intensities were assigned a value of 1 . If the value of all the samples tested was 1 , those cytokines were removed from further analysis. The mean and the standard error of each cytokine were calculated separately.

Histological staining. The skin specimens were embedded in paraffin blocks after they had been fixed in $4 \%$ paraformaldehyde solution (Beyotime Institute of Biotechnology). Sections of $5 \mu \mathrm{m}$ were deparaffinized and stained using the hematoxylin and eosin (H\&E; Beyotime Institute of Biotechnology) staining method. The skin specimens were examined and evaluated in a blinded manner under a standard light microscope (CX31; Olympus Corporation, Tokyo, Japan).

DAPI fluorescence staining. Sections (5- $\mu \mathrm{m}$ thickness) were obtained, deparaffinized and stained with DAPI solution (Beyotime Institute of Biotechnology) at room temperature for 3-5 min, washed three times using PBS and observed with a fluorescence microscope.

Subcutaneous implantation of skin matrix. ADM and DDBDM were trimmed to $1 \times 1-\mathrm{cm}$ sections and implanted subcutaneously into 48 mice. Briefly, following anesthetization by intraperitoneal injection with $10 \%$ chloral hydrate $(0.3 \mathrm{ml} / \mathrm{kg})$, the fur on the dorsum of the mice was shaved, a vertical incision was made in the middle of the dorsum and the skin was separated from the subcutaneous tissue by a blunt dissection in order to create two $15 \mathrm{~mm}$-deep subcutaneous bursae. Subsequently, the ADM was implanted in the left bursa and the DDBDM in the right. The incision was closed and the gap in the subcutaneous tissue was sutured to prevent the ADM and DDBDM from touching. The wound was disinfected using $1 \%$ povidone iodine solution every day. At 1, 2, 3 and 4 weeks of subcutaneous implantation, three mice were randomly selected for sacrifice by decapitation, prior to immersion in $75 \%$ alcohol for $3 \mathrm{~min}$. Subsequently, the dorsal skins of the mice were cut open and separated from the subcutaneous tissue in order to expose the implanted skin matrices, which were then stained using H\&E and DAPI. Images of the skin matrices were captured under a microscope (BX53; Olympus Corporation).

Statistical analysis. All data are presented as the mean \pm standard deviation. Dual comparisons between groups were evaluated with the Student's t-test, using the SPSS software, version 19.0 (IBM SPSS, Armonk, NY, USA). P<0.05 was considered to indicate a statistically significant difference.

\section{Results}

DDBDM preparation and its biological properties. Images of ADM and DDBDM prior to implantation are presented in 
Table II. Expression values of cytokines with significant differences between burned and normal skin samples.

\begin{tabular}{|c|c|c|c|c|c|c|c|c|}
\hline Cytokine & $\begin{array}{c}\text { Normal } \\
\text { skin }^{\mathrm{a}}\end{array}$ & $\begin{array}{c}\text { Burned } \\
\text { skin }^{\mathrm{a}}\end{array}$ & P-value & $\begin{array}{c}\text { Fold } \\
\text { change }\end{array}$ & $\mathrm{ADM}^{\mathrm{a}}$ & DDBDM $^{\mathrm{a}}$ & P-value & $\begin{array}{c}\text { Fold } \\
\text { change }\end{array}$ \\
\hline Activin $\beta C$ & $393.5 \pm 61.5$ & $1.0 \pm 0.0$ & $<0.01$ & 393.500 & $76.5 \pm 0.5$ & $34.0 \pm 3.0$ & $<0.01$ & 2.250 \\
\hline 6Ckine & $193.5 \pm 18.5$ & $1.0 \pm 0.0$ & $<0.01$ & 193.500 & $49.0 \pm 12.0$ & $5.5 \pm 0.5$ & $<0.01$ & 8.909 \\
\hline Decorin & $49.0 \pm 11.0$ & $1.0 \pm 0.0$ & $<0.01$ & 49.000 & $913.5 \pm 76.5$ & $180.0 \pm 6.0$ & $<0.01$ & 5.075 \\
\hline IL-10 & $1,460.0 \pm 94.0$ & $336.0 \pm 8.0$ & $<0.01$ & 4.345 & $221.0 \pm 7.0$ & $98.0 \pm 1.0$ & $<0.01$ & 2.255 \\
\hline IL-17 & $1,057.5 \pm 46.5$ & $268.5 \pm 60.5$ & $<0.01$ & 3.939 & $180.0 \pm 8.0$ & $107.5 \pm 3.5$ & $<0.01$ & 1.674 \\
\hline MMP-14 & $929.0 \pm 37.0$ & $372.0 \pm 15.0$ & $<0.01$ & 2.497 & $162.0 \pm 2.0$ & $81.0 \pm 7.0$ & $<0.01$ & 2.000 \\
\hline HVEM & $1,193.5 \pm 118.5$ & $499.0 \pm 212.0$ & $<0.01$ & 2.392 & $219.5 \pm 1.5$ & $68.5 \pm 1.5$ & $<0.01$ & 3.204 \\
\hline TNF- $\alpha$ & $1,327.0 \pm 32.0$ & $637.5 \pm 18.5$ & $<0.01$ & 2.082 & $184.0 \pm 17.0$ & $107.0 \pm 4.0$ & $<0.01$ & 1.720 \\
\hline IL-3 & $2,519.0 \pm 33.0$ & $1,356.5 \pm 56.5$ & $<0.01$ & 1.857 & $303.0 \pm 15.0$ & $145.5 \pm 3.5$ & $<0.01$ & 2.082 \\
\hline TGF- $\beta 1$ & $1,205.5 \pm 111.5$ & $688.5 \pm 39.5$ & $<0.01$ & 1.751 & $212.0 \pm 22.0$ & $115.5 \pm 4.5$ & $<0.01$ & 1.835 \\
\hline RELM $\beta$ & $139.0 \pm 3.0$ & $562.0 \pm 28.0$ & $<0.01$ & 0.247 & $41.5 \pm 0.5$ & $75.0 \pm 1.0$ & $<0.01$ & 0.553 \\
\hline IL-9 & $148.5 \pm 60.5$ & $921.5 \pm 267.5$ & $<0.01$ & 0.161 & $65.5 \pm 9.5$ & $258.0 \pm 32.0$ & $<0.01$ & 0.254 \\
\hline IL-23 R & $12.8 \pm 13.2$ & $157.5 \pm 46.5$ & $<0.01$ & 0.081 & $2.5 \pm 2.2$ & $14.5 \pm 4.5$ & $<0.05$ & 0.172 \\
\hline Thrombospondin & $20.5 \pm 26.0$ & $907.0 \pm 46.0$ & $<0.01$ & 0.023 & $25.5 \pm 14.5$ & $65.5 \pm 9.5$ & $<0.05$ & 0.389 \\
\hline
\end{tabular}

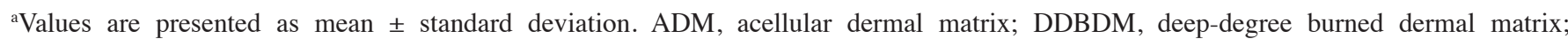
6Ckine, Chemokine (C-C motif) ligand 21; IL, interleukin; MMP, matrix matelloproteinase; HVEM, herpesvirus entry mediator; TNF- $\alpha$, tumor necrosis factor- $\alpha$; TGF- $\beta 1$, transforming growth factor- $\beta 1$; RELM $\beta$, resistin-like molecule $\beta$; R, receptor.

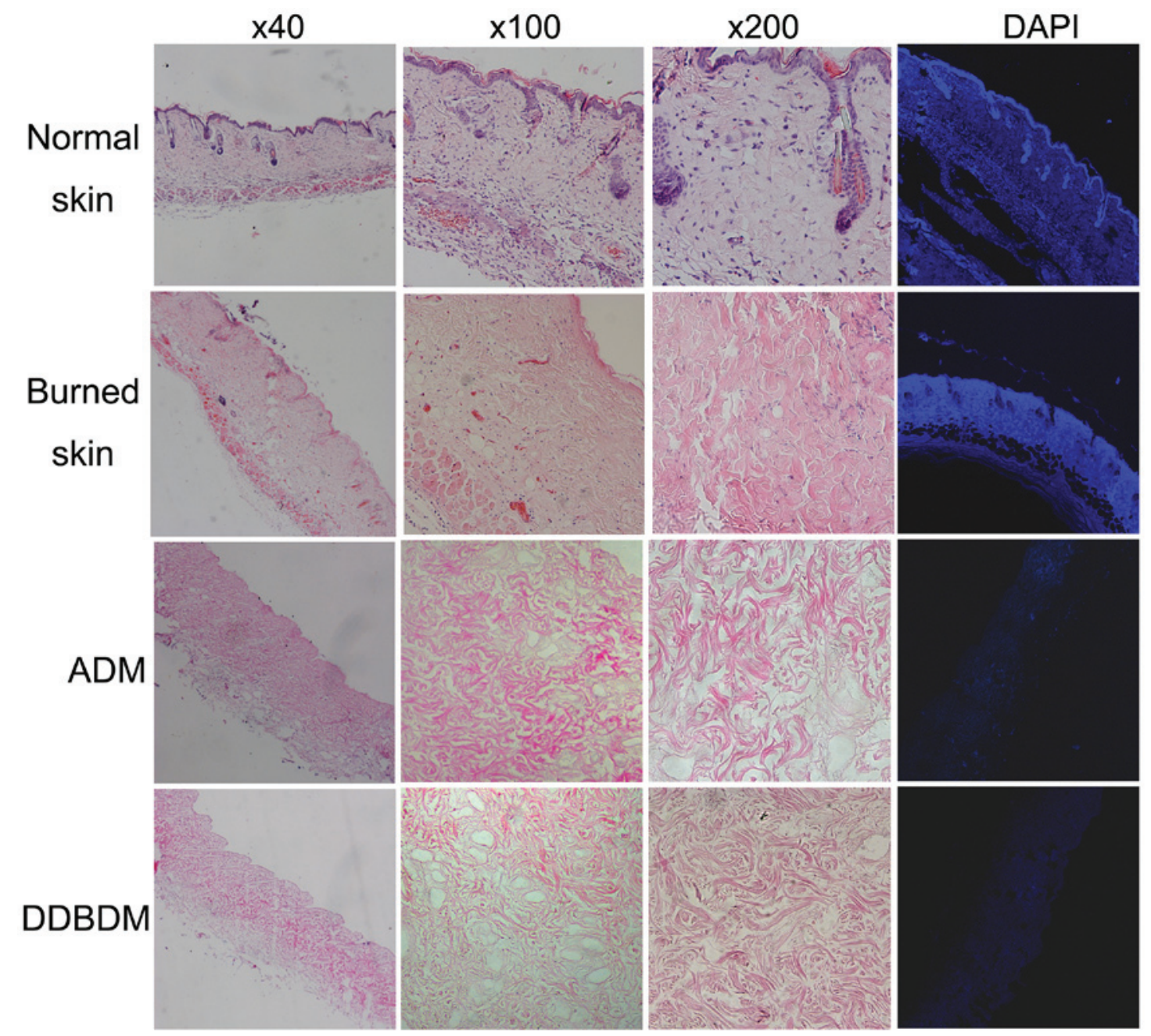

Figure 2. Hematoxylin and eosin and DAPI staining of normal skin, burned skin, ADM and DDBDM sections at various magnifications. ADM, acellular dermal matrix; DDBDM, deep-degree burned dermal matrix. 
A

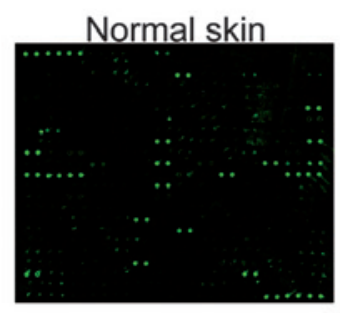

Burned skin

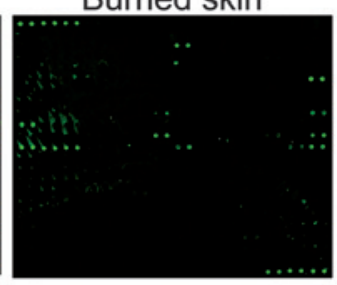

ADM

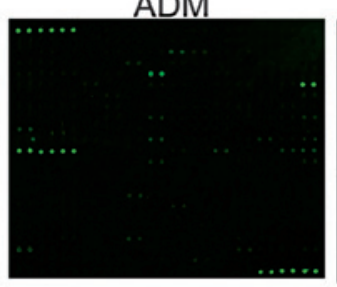

DDBDM

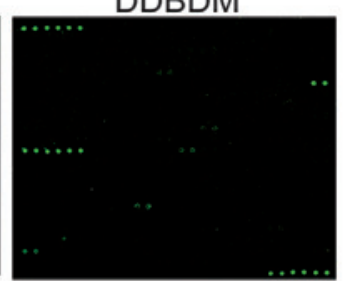

B

\begin{tabular}{|c|c|c|c|c|c|c|c|c|c|c|c|c|c|c|}
\hline & 1 & 2 & 3 & 4 & 5 & 6 & 7 & 8 & 9 & 10 & 11 & 12 & 13 & 14 \\
\hline & $\begin{array}{c}\text { Positive } \\
1\end{array}$ & $\begin{array}{c}\text { Positive } \\
1\end{array}$ & $\begin{array}{l}\text { Positive } \\
2\end{array}$ & $\begin{array}{c}\text { Positive } \\
2\end{array}$ & $\begin{array}{c}\text { Positive } \\
3\end{array}$ & $\begin{array}{c}\text { Positive } \\
3\end{array}$ & neg & neg & 6Ckine & 6Ckine & Activin A & Activin A & Activin C & Activin C \\
\hline ? & Artemin & Artemin & Axl & Axl & b FGF & b FGF & CD80 & CD80 & BAFF R & BAFF R & BCMA & BCMA & $\beta$-Catenin & $\beta$-Catenin \\
\hline 3 & CCL8 & CCL8 & CCR10 & CCR10 & CCR3 & CCR3 & CCR4 & CCR4 & CCR6 & CCR6 & CCR7 & CCR7 & CCR9 & CCR9 \\
\hline 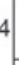 & CD40 & CD40 & $\begin{array}{l}\text { CD40 } \\
\text { Ligand }\end{array}$ & $\begin{array}{l}\text { CD40 } \\
\text { Ligand }\end{array}$ & $\begin{array}{c}\text { Cerberus } \\
1\end{array}$ & $\begin{array}{c}\text { Cerberus } \\
1\end{array}$ & $\begin{array}{c}\text { Chordin- } \\
\text { Like 2 }\end{array}$ & $\begin{array}{c}\text { Chordin- } \\
\text { Like 2 }\end{array}$ & $\begin{array}{l}\text { Tissue } \\
\text { Factor }\end{array}$ & $\begin{array}{l}\text { Tissue } \\
\text { Factor }\end{array}$ & IL-2 RY & IL-2 RY & CRG-2 & CRG-2 \\
\hline 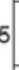 & CXCL16 & CXCL16 & $\begin{array}{l}\text { CXCR2 I } \\
\text { IL-8 RB }\end{array}$ & $\begin{array}{l}\text { CXCR2 I } \\
\text { IL-8 RB }\end{array}$ & CXCR3 & CXCR3 & CXCR4 & CXCR4 & CXCR6 & CXCR6 & DAN & DAN & Decorin & Decorin \\
\hline 6 & EGF R & EGF R & $\begin{array}{l}\text { EG- } \\
\text { VEGF }\end{array}$ & $\begin{array}{l}\text { EG- } \\
\text { VEGF }\end{array}$ & Endocan & Endocan & Endoglin & Endoglin & Endostatin & Endostatin & Eotaxin & Eotaxin & Eotaxin-2 & Eotaxin-2 \\
\hline & $\begin{array}{c}\text { Fas } \\
\text { Ligand }\end{array}$ & $\begin{array}{c}\text { Fas } \\
\text { Ligand }\end{array}$ & CD32b & CD32b & FGF R3 & FGF R3 & FGF R4 & FGF R4 & FGF R5 $\beta$ & FGF R5 $\beta$ & FGF-21 & FGF-21 & $\begin{array}{c}\text { Fit-3 } \\
\text { Ligand }\end{array}$ & $\begin{array}{c}\text { Fit-3 } \\
\text { Ligand }\end{array}$ \\
\hline $8 \mid$ & G-CSF & G-CSF & GDF-1 & GDF-1 & GDF-3 & GDF-3 & GDF-5 & GDF-5 & GDF-8 & GDF-8 & GDF-9 & GDF-9 & GFR $\alpha-2$ & GFR $\alpha-2$ \\
\hline 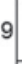 & $\begin{array}{c}\text { Granzyme } \\
\text { D }\end{array}$ & $\begin{array}{c}\text { Granzyme } \\
\text { D }\end{array}$ & $\begin{array}{c}\text { Granzyme } \\
G\end{array}$ & $\begin{array}{c}\text { Granzyme } \\
G\end{array}$ & Gremlin & Gremlin & \begin{tabular}{|c|} 
Growth \\
Hormone R
\end{tabular} & $\begin{array}{c}\text { Growth } \\
\text { Hormone R }\end{array}$ & HGF R & GF R & HGF & HGF & \begin{tabular}{|c|} 
TNFRSF \\
14
\end{tabular} & $\begin{array}{c}\text { TNFRSF } \\
14\end{array}$ \\
\hline & IFN- $\gamma$ & IFN- $y$ & IFN-y R1 & IFN-y R1 & IGFBP-1 & IGFBP-1 & IGFBP-2 & IGFBP-2 & IGFBP-3 & IGFBP-3 & IGFBP-5 & IGFBP-5 & IGFBP-6 & IGFBP-6 \\
\hline & IL-1 R9 & IL-1 R9 & IL-1 R I & IL-1 R I & IL-1 R II & IL-1 R II & IL-2 & IL-2 & IL-2 Ra & IL-2 Ra & $\mathrm{IL}-2 \mathrm{R} \beta$ & IL-2 R $\beta$ & IL-3 & IL-3 \\
\hline
\end{tabular}

C

\begin{tabular}{|c|c|c|c|c|c|c|c|c|c|c|c|c|c|c|}
\hline & 15 & 16 & 17 & 18 & 19 & 20 & 21 & 22 & 23 & 24 & 25 & 26 & 27 & 28 \\
\hline & $\begin{array}{c}\text { Activin } \\
\text { RIB }\end{array}$ & $\begin{array}{c}\text { Activin } \\
\text { RIB } \\
\end{array}$ & Acrp30 & Acrp30 & AgRP & AgRP & ALCAM & ALCAM & $\begin{array}{c}\text { Anglopoietin- } \\
\text { like } 2\end{array}$ & \begin{tabular}{|c} 
Angiopoietin- \\
like 2
\end{tabular} & $\begin{array}{c}\text { Angiopoletin- } \\
\text { like } 3\end{array}$ & $\begin{array}{c}\text { Angiopoietin- } \\
\text { like } 3\end{array}$ & AR & AR \\
\hline 2 & BLC & BLC & BTC & BTC & $\begin{array}{c}\text { Cardiotro } \\
\text { phin-1 }\end{array}$ & $\begin{array}{c}\text { Cardiotro } \\
\text { phin-1 }\end{array}$ & CCL1 & CCL1 & CCL28 & CCL28 & CCL4 & CCL4 & CCL7 & CCL7 \\
\hline 3 & CD11b & CD11b & CD14 & CD14 & CRP & CRP & CD27 & CD27 & $\begin{array}{l}\text { CD27 } \\
\text { Ligand }\end{array}$ & $\begin{array}{l}\text { CD27 } \\
\text { Ligand }\end{array}$ & CD30 & CD30 & CD30 L & CD30 L \\
\hline 4 & Cripto & Cripto & $\begin{array}{c}\begin{array}{c}\text { Crossvein } \\
\text { less-2 }\end{array} \\
\end{array}$ & $\begin{array}{c}\text { Crossvein } \\
\text { less-2 }\end{array}$ & Cryptic & Cryptic & Csk & Csk & CTACK & CTACK & CTLA-4 & CTLA-4 & CXCL14 & CXCL14 \\
\hline 5 & DKK-1 & DKK-1 & DKK-3 & DKK-3 & DKK-4 & DKK-4 & DPPIV & DPPIV & DR3 & DR3 & Dtk & Dtk & EDAR & EDAR \\
\hline 3 & Epigen & Epigen & $\begin{array}{c}\text { Epireg- } \\
\text { ulin }\end{array}$ & $\begin{array}{c}\text { Epireg- } \\
\text { ulin }\end{array}$ & EPO & EPO & E-Selectin & E-Selectin & FADD & FADD & FAM3B & FAM3B & Fas & Fas \\
\hline 7 & FLRG & FLRG & $\begin{array}{c}\text { Follistatin } \\
\text {-like } 1 \\
\end{array}$ & $\begin{array}{c}\text { Follistatin } \\
\text {-like } 1\end{array}$ & $\begin{array}{c}\text { Fractal- } \\
\text { kine }\end{array}$ & $\begin{array}{c}\begin{array}{c}\text { Fractal- } \\
\text { kine }\end{array} \\
\end{array}$ & Frizzled-1 & Frizzled-1 & Frizzled-6 & Frizzled-6 & Frizzled-7 & Frizzled-7 & Galectin-3 & Galectin-3 \\
\hline 3 & GFR a-3 & GFR $\alpha-3$ & GFR $\alpha-4$ & GFR $a-4$ & TR & $R$ & $\begin{array}{c}\text { GITR } \\
\text { Ligand }\end{array}$ & $\begin{array}{c}\text { GITR } \\
\text { Ligand }\end{array}$ & 2 & lut2 & GM-CSF & GM-CSF & $\begin{array}{c}\text { Granzym } \\
\text { e B }\end{array}$ & $\begin{array}{c}\text { Granzym } \\
\text { e B }\end{array}$ \\
\hline 9 & ICAM-1 & ICAM-1 & ICAM-2 & ICAM-2 & ICAM-5 & ICAM-5 & ICK & ICK & $\begin{array}{c}\mathrm{IFN}-\alpha / \beta \\
\mathrm{R} 1\end{array}$ & $\begin{array}{c}\text { IFN- } \alpha / \beta \\
\text { R1 }\end{array}$ & $\begin{array}{c}\mathrm{IFN}-\alpha / \beta \\
\mathrm{R} 2\end{array}$ & $\begin{array}{c}\mid \mathrm{FN}-\alpha / \beta \\
\mathrm{R} 2\end{array}$ & IFN- $\beta$ & IFN- $\beta$ \\
\hline & IGFBP-7 & IGFBP-7 & IGF- I & IGF- I & IGF-II & IGF-II & IL-1 $\alpha$ & IL-1 $1 \alpha$ & IL-1 $1 \beta$ & IL-1 $\beta$ & IL-1 R4 & IL-1 R4 & IL-1 R6 & IL-1 R6 \\
\hline & IL-3 Ra & IL-3 Ra & IL-3 R $\beta$ & IL-3 R $\beta$ & IL-4 & IL-4 & IL-4 R & IL-4 R & IL-5 & IL-5 & IL-5 Ra & IL-5 Ra & IL-6 & IL-6 \\
\hline
\end{tabular}

Figure 3. Biotinylated antibody cytokine microarray assay. (A) Images of the biotinylated antibody arrays. (B and C) Grid map of cytokines from the biotinylated antibody arrays.

Fig. 1. The ADM and DDBDM were white in color, and had good elasticity and tenacity. However, the DDBDM was softer and thinner than ADM. The physical properties of ADM and DDBDM are presented in Table I. There was a statistically significant difference between ADM and DDBDM in each of the properties measured $(\mathrm{P}<0.05)$.

Under microscopic observation, normal skin samples exhibited an integrated structure, including epidermis, dermis and appendages. DAPI staining demonstrated the presence of high DNA levels in the nuclei of the normal skin. By contrast, collagen fibers in the burned skin samples were thickened and swollen, coagulated necrosis was observed, and the number of integrated nuclei decreased. DAPI staining demonstrated that the DNA was diffusely distributed due to karyorrhexis and karyolysis in the burned skin. No DNA was observed in ADM and DDBDM samples as they underwent acellular disposal. 
D

\begin{tabular}{|c|c|c|c|c|c|c|c|c|c|c|c|c|c|c|}
\hline & 1 & 2 & 3 & 4 & 5 & 6 & 7 & 8 & 9 & 10 & 11 & 12 & 13 & 14 \\
\hline 12 & \begin{tabular}{|c|} 
Positive \\
1
\end{tabular} & \begin{tabular}{|c|} 
Positive \\
1
\end{tabular} & $\begin{array}{l}\text { Positive } \\
2\end{array}$ & \begin{tabular}{|c|} 
Positive \\
2
\end{tabular} & \begin{tabular}{|c|} 
Positive \\
3
\end{tabular} & \begin{tabular}{|c|} 
Positive \\
3
\end{tabular} & neg & neg & IL-6 R & IL-6 R & IL-7 & IL-7 & IL-7 Ra & IL-7 Ra \\
\hline 13 & $\mid \mathrm{L}-12 \mathrm{R} \beta 1$ & IL-12 Rß1 & IL-13 & IL-13 & IL-13 Ra2 & IL-13 Ra2 & IL-15 & IL-15 & IL-15 Ra & IL-15 Ra & IL-16 & IL-16 & IL-17 & IL-17 \\
\hline 4 & IL-17RD & IL-17RD & IL-18 Ra & IL-18 Ra & IL-20 & IL-20 & IL-20 Ra & IL-20 Ra & IL-21 & IL-21 & IL-21 R & IL-21 R & IL-22 & IL-22 \\
\hline 5 & IL-31 RA & IL-31 RA & Insulin & Insulin & CD18 & CD18 & I-TAC & I-TAC & KC & $\mathrm{KC}$ & Kremen-1 & Kremen-1 & Kremen-2 & Kremen-2 \\
\hline 6 & L-Selectin & L-Selectin & Lungkine & Lungkine & $\begin{array}{c}\text { Lymphot } \\
\text { actin }\end{array}$ & $\begin{array}{c}\text { Lymphot } \\
\text { actin }\end{array}$ & $\begin{array}{c}\text { Lymphot } \\
\text { oxin } \beta R \\
\end{array}$ & $\begin{array}{c}\text { Lymphot } \\
\text { oxin } \beta R\end{array}$ & MadCAM-1 & MAdCAM-1 & MCP-1 & ACP-1 & MCP-5 & MCP-5 \\
\hline 17 & MIP-2 & MIP-2 & MIP-3a & MIP-3a & MIP-3 $\beta$ & MIP-3 $\beta$ & MMP-2 & MMP-2 & MMP-3 & MMP-3 & MMP-9 & MMP-9 & MMP-12 & MMP-12 \\
\hline 18 & \begin{tabular}{|c|}
$\begin{array}{c}\text { Osteopo } \\
\text { ntin }\end{array}$ \\
\end{tabular} & $\begin{array}{c}\text { Osteopo } \\
\text { ntin }\end{array}$ & $\begin{array}{l}\text { Osteopor } \\
\text { otegerin }\end{array}$ & \begin{tabular}{|l|}
$\begin{array}{l}\text { Osteopor } \\
\text { otegerin }\end{array}$ \\
\end{tabular} & $\begin{array}{l}\text { OX40 } \\
\text { Ligand }\end{array}$ & $\begin{array}{l}\text { OX40 } \\
\text { Ligand }\end{array}$ & PDGF C & PDGF C & PDGF Ra & PDGF Ra & PDGF R $\beta$ & PDGF R $\beta$ & Pentraxin3 & Pentraxin3 \\
\hline 19 & RELM $\beta$ & RELM $\beta$ & Resistin & Resistin & S100A10 & S100A10 & SCF & SCF & SCF R & SCF R & SDF-1 & SDF-1 & $\begin{array}{c}\text { Serum } \\
\text { Amyloid A1 }\end{array}$ & $\begin{array}{l}\text { Serum } \\
\text { Amyloid A1 }\end{array}$ \\
\hline 20 & TARC & TARC & TCA-3 & TCA-3 & TCCR & TCCR & TECK & TECK & TFPI & TFPI & TGF- $\beta 1$ & TGF- $\beta 1$ & TGF- $\beta 2$ & TGF- $\beta 2$ \\
\hline 21 & TIMP-2 & TIMP-2 & TIMP-4 & TIMP-4 & TL1A & TL1A & TLR1 & TLR1 & TLR2 & TLR2 & TLR3 & TLR3 & TLR4 & TLR4 \\
\hline 2 & $\begin{array}{c}\text { TRAIL } \\
\text { R2 }\end{array}$ & $\begin{array}{c}\text { TRAIL } \\
\text { R2 } \\
\end{array}$ & TRANCE & TRANCE & TREM-1 & TREM-1 & TROY & TROY & TSLP & TSLP & TSLP R & TSLP R & TWEAK & TWEAK \\
\hline & VEGF R1 & VEGF R1 & VEGF R2 & VEGF R2 & VEGF R3 & VEGF R3 & VEGF-B & VEGF-B & VEGFC & VEGFC & VEGF-D & VEGF-D & WIF-1 & WIF-1 \\
\hline
\end{tabular}

$\mathbf{E}$

\begin{tabular}{|c|c|c|c|c|c|c|c|c|c|c|c|c|c|c|}
\hline & 15 & 16 & 17 & 18 & 19 & 20 & 21 & 22 & 23 & 24 & 25 & 26 & 27 & 28 \\
\hline & IL-9 & IL-9 & IL-9 R & IL-9 R & IL-10 & IL-10 & IL-10 Ra & IL-10 Ra & IL-11 & IL-11 & \begin{tabular}{|c|} 
IL-12 \\
p40/p70
\end{tabular} & \begin{tabular}{c|} 
IL-12 \\
p40/p70
\end{tabular} & IL-12 p70 & IL-12 p70 \\
\hline & IL-17BR & IL-17BR & IL-17C & IL-17C & IL-17D & IL-17D & IL-17E & IL-17E & IL-17F & IL-17F & IL-17R & IL-17R & IL-17RC & IL-17RC \\
\hline & IL-22BP & IL-22BP & IL-23 & IL-23 & IL-23 R & IL-23 R & IL-24 & IL-24 & IL-27 & IL-27 & IL-28 & IL-28 & IL-31 & IL-31 \\
\hline & Lefty-1 & Lefty-1 & Leptin R & Leptin R & $\begin{array}{c}\text { LEPTIN } \\
(\mathrm{OB})\end{array}$ & $\begin{array}{c}\text { LEPTIN } \\
(\mathrm{OB})\end{array}$ & LIF & LIF & LIGHT & LIGHT & LIX & LIX & LRP-6 & LRP-6 \\
\hline & M-CSF & M-CSF & MDC & MDC & MFG-E8 & MFG-E8 & MFRP & MFRP & MIG & MIG & MIP-1 $1 \alpha$ & MIP-1 $1 \alpha$ & MIP-1Y & MIP-1Y \\
\hline & MMP-14 & MMP-14 & MMP-24 & MMP-24 & $\begin{array}{c}\text { Neuregul } \\
\text { in-3 }\end{array}$ & $\begin{array}{c}\text { Neuregul } \\
\text { in-3 }\end{array}$ & Neurturin & Neurturin & NGF R & NGF R & NOV & NOV & GPNMB & GPNMB \\
\hline & PF-4 & PF-4 & PIGF-2 & PIGF-2 & $\begin{array}{l}\text { Progranu } \\
\text { lin }\end{array}$ & $\begin{array}{l}\text { Progranu } \\
\text { lin }\end{array}$ & Prolactin & Prolactin & P-Selectin & P-Selectin & RAGE & RAGE & RANTES & RANTES \\
\hline & Shh-N & Shh-N & SIGIRR & SIGIRR & SLPI & SLPI & Soggy-1 & Soggy-1 & SPARC & SPARC & $\begin{array}{c}\text { Spinesin } \\
\text { Ectodomain }\end{array}$ & $\begin{array}{c}\text { Spinesin } \\
\text { Ectodomain }\end{array}$ & TACI & TACI \\
\hline & TGF- $\beta 3$ & TGF- $\beta 3$ & $F-\beta R I$ & TGF- $\beta R$ I & TGF- $\beta$ R II & TGF- $\beta$ R II & \begin{tabular}{|c}
$\begin{array}{c}\text { Thrombo } \\
\text { spondin }\end{array}$ \\
\end{tabular} & \begin{tabular}{|c|}
$\begin{array}{c}\text { Thrombo } \\
\text { spondin }\end{array}$ \\
\end{tabular} & $\begin{array}{c}\text { Thymus } \\
\text { Chemokine-1 }\end{array}$ & $\begin{array}{c}\text { Thymus } \\
\text { Chemokine-1 }\end{array}$ & Tie-2 & Tie-2 & TIMP-1 & TIMP-1 \\
\hline & TMEFF1 & TMEFF1 & TNF R I & TNF R I & TNF R II & TNF R II & TNF- $\alpha$ & TNF- $\alpha$ & TNF- $\beta$ & TNF- $\beta$ & TPO & TPO & TRAIL & TRAIL \\
\hline & TWEAK R & TWEAK R & Ubiqultin & Ubiqultin & uPAR & UPAR & Urokinase & Urokinase & VCAM-1 & VCAM-1 & \begin{tabular}{|c} 
VE- \\
Cadherin
\end{tabular} & \begin{tabular}{|c|} 
VE- \\
Cadherin \\
\end{tabular} & VEGF & VEGF \\
\hline & WISP-1 & WISP-1 & Neg & Neg & Neg & leg & Neg & Neg & $\begin{array}{c}\text { Positive } \\
3\end{array}$ & $\begin{array}{c}\text { Positive } \\
3\end{array}$ & \begin{tabular}{|c} 
Positive \\
2
\end{tabular} & $\begin{array}{c}\text { Positive } \\
2\end{array}$ & $\begin{array}{c}\text { Positive } \\
1\end{array}$ & $\begin{array}{c}\text { Positive } \\
1\end{array}$ \\
\hline
\end{tabular}

Figure 3. Continued. (D and E) Grid map of cytokine names from the biotinylated antibody microarrays.

$\mathrm{H} \& \mathrm{E}$ staining demonstrated that the collagens in the ADM were loose but well-organized, and no residual cellular nuclei were observed. In the DDBDM, the collagens were thickened, disorganized and fractured; however, the reticulate structure was maintained (Fig. 2).

Cytokine analysis using biotinylated antibody microarrays. Images of the biotinylated antibody arrays are presented in Fig. 3A, and the names of all cytokines analyzed are presented in Fig. 3B-E. If expression of a cytokine was negative in all samples, it was eliminated from further analysis. Subsequently, 227 of the 308 cytokines remained for analysis. The values from the two groups were compared. The following conditions were considered to be significant: i) A fold change in cytokine level of $<0.66$ or $>1.5$; ii) at least one value $>200$ and iii) $\mathrm{P}<0.05$.
Effects of burn on the expression level of cytokines. To explore whether the expression of cytokines is affected by burn, the values of cytokine expression were compared between normal and burned skin, and between ADM and DDBDM. If the results demonstrated a significant increase or decrease in both comparisons of the same cytokine, the cytokine was considered to be affected by burn. Results showed that the expression of 14 cytokines were altered by burn (Table II).

Effects of cell extraction on the expression level of cytokines. The expression levels of cytokines were compared between the normal skin and ADM, and between burned skin and DDBDM. If the results demonstrated a significant increase or decrease in both comparisons of the same cytokine, the cytokine was then considered to be affected by acellular treatment. Results 


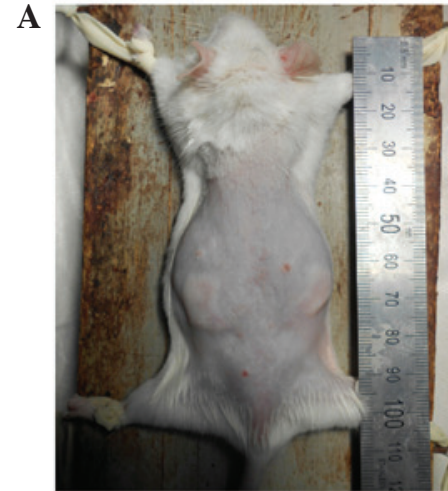

1 week

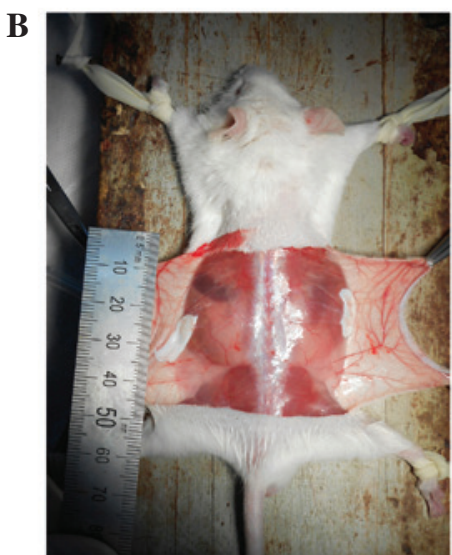

1 week

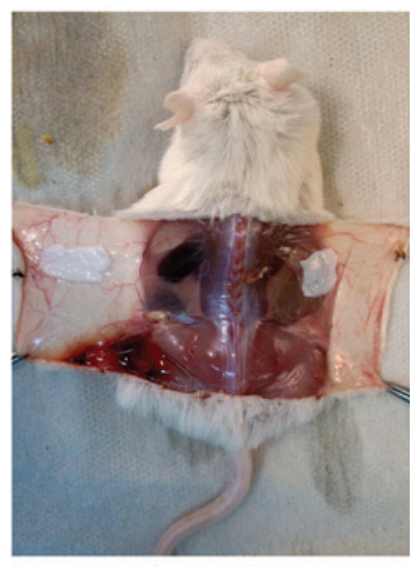

2 weeks

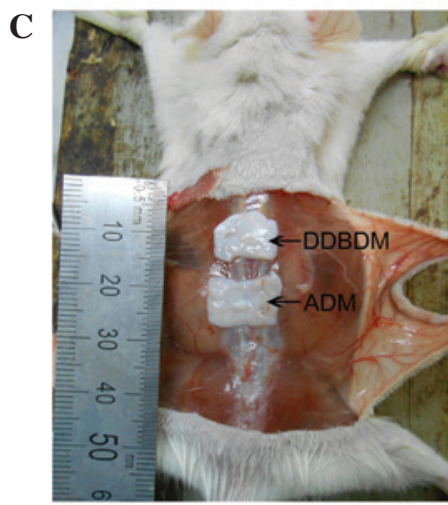

1 week

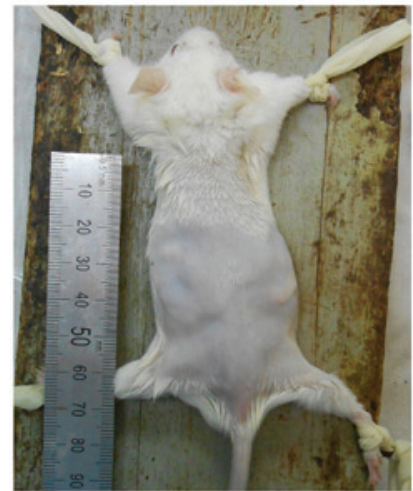

4 weeks

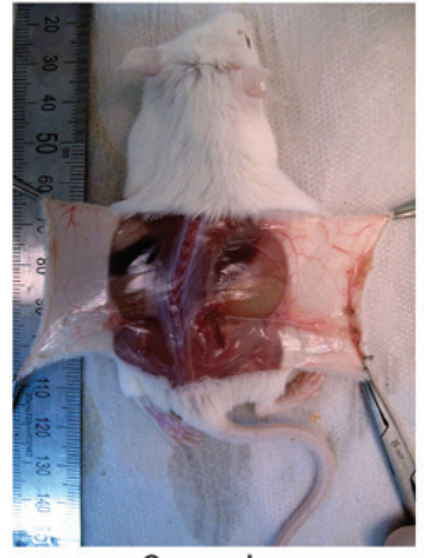

3 weeks

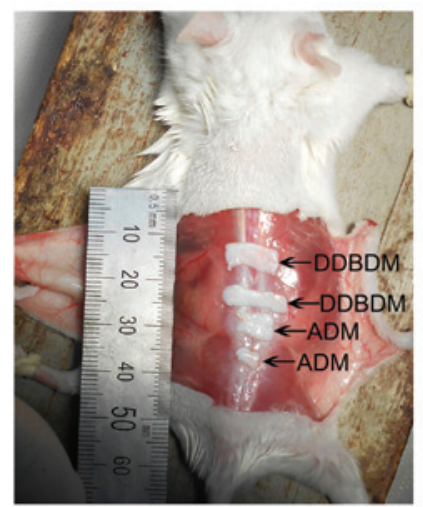

4 weeks

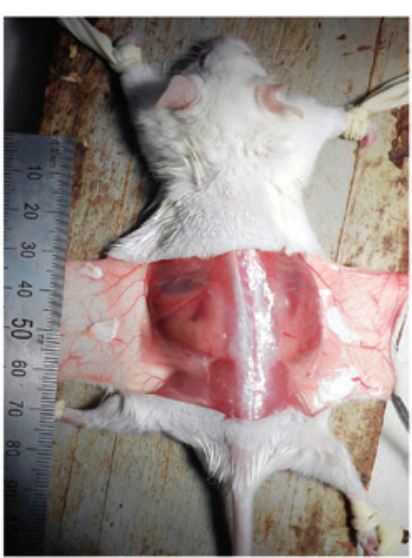

4 weeks

Figure 4. General observations following subcutaneous implantation of the ADM and DDBDM. (A) The healed wound 1 and 4 weeks after subcutaneous implantation. (B) Images of the subcutaneously implanted ADM (left) and DDBDM (right) after 1,2,3 and 4 weeks. (C) Comparison of the ADM and DDBDM after they were separated from the skin. ADM, acellular dermal matrix; DDBDM, deep-degree burned dermal matrix.

showed that the levels of 23 cytokines were affected by cell extraction (Table III).

Differences in cytokine expression between ADM and $D D B D M$. The expression levels of cytokines in ADM and DDBDM were compared. The results are indicated in Table IV. The levels of 17 cytokines were decreased in DDBDM compared with ADM, however IL-9 and MMP-9 levels were increased.
General observation of the ADM and DDBDM following subcutaneous implantation. Following subcutaneous implantation, the skin wounds of the mice healed well (Fig. 4A). Four weeks after implantation, ADM and DDBDM degraded incompletely and maintained the some integrity (Fig. 4B and C). No marked inflammatory reaction or rejection was observed, demonstrating histocompatibility of ADM and DDBDM. After one week, the ADM and DDBDM could be easily separated from the subcutaneous tissue (Fig. 4B and C). Two weeks 
Table III. Expression values of the cytokines with significant differences following cell extraction.

\begin{tabular}{|c|c|c|c|c|c|c|c|c|}
\hline Cytokine & $\begin{array}{c}\text { Normal } \\
\text { skin }^{\mathrm{a}}\end{array}$ & $\mathrm{ADM}^{\mathrm{a}}$ & P-value & $\begin{array}{c}\text { Fold } \\
\text { change }\end{array}$ & $\begin{array}{l}\text { Burned } \\
\text { skin }^{\mathrm{a}}\end{array}$ & DDBDM $^{\mathrm{a}}$ & P-value & $\begin{array}{c}\text { Fold } \\
\text { change }\end{array}$ \\
\hline RANTES & $115.0 \pm 7.0$ & $9.0 \pm 7.0$ & $<0.01$ & 12.778 & $208.5 \pm 102.5$ & $19.0 \pm 10.0$ & $<0.05$ & 10.974 \\
\hline IL-6 & $2,195.0 \pm 32.0$ & $226.0 \pm 20.0$ & $<0.01$ & 9.712 & $3,129.0 \pm 4.0$ & $131.5 \pm 9.5$ & $<0.01$ & 23.795 \\
\hline IL-3 & $2,519.0 \pm 33.0$ & $303.0 \pm 15.0$ & $<0.01$ & 8.314 & $1,356.5 \pm 56.5$ & $145.5 \pm 3.5$ & $<0.01$ & 9.323 \\
\hline TNF- $\alpha$ & $1,327.0 \pm 32.0$ & $184.0 \pm 17.0$ & $<0.01$ & 7.212 & $637.5 \pm 18.5$ & $107.0 \pm 4.0$ & $<0.01$ & 5.958 \\
\hline IL-10 & $1,460.0 \pm 94.0$ & $221.0 \pm 7.0$ & $<0.01$ & 6.606 & $336.0 \pm 8.0$ & $98.0 \pm 1.0$ & $<0.01$ & 3.429 \\
\hline GDF-1 & $316.5 \pm 6.5$ & $50.5 \pm 14.5$ & $<0.01$ & 6.267 & $155.5 \pm 23.5$ & $53.0 \pm 1.0$ & $<0.01$ & 2.934 \\
\hline IL-12 p70 & $2,000.5 \pm 120.5$ & $322.0 \pm 11.0$ & $<0.01$ & 6.213 & $1,984.5 \pm 21.5$ & $150.0 \pm 16.0$ & $<0.01$ & 13.230 \\
\hline IFN- $\beta$ & $1,117.5 \pm 6.5$ & $182.0 \pm 9.0$ & $<0.01$ & 6.140 & $1,252.0 \pm 85.0$ & $136.0 \pm 5.0$ & $<0.01$ & 9.206 \\
\hline MCP-1 & $1,125.0 \pm 20.0$ & $187.5 \pm 12.5$ & $<0.01$ & 6.000 & $829.5 \pm 89.5$ & $89.5 \pm 0.5$ & $<0.01$ & 9.268 \\
\hline IFN- $\gamma$ & $1,990.5 \pm 81.5$ & $332.5 \pm 6.5$ & $<0.01$ & 5.986 & $1,357.5 \pm 75.5$ & $163.0 \pm 1.0$ & $<0.01$ & 8.328 \\
\hline CD11b & $2,094.0 \pm 231.0$ & $355.5 \pm 4.5$ & $<0.01$ & 5.890 & $1,527.0 \pm 80.0$ & $152.0 \pm 6.0$ & $<0.01$ & 10.046 \\
\hline IL-17 & $1,057.5 \pm 46.5$ & $180.0 \pm 8.0$ & $<0.01$ & 5.875 & $268.5 \pm 60.5$ & $107.5 \pm 3.5$ & $<0.05$ & 2.498 \\
\hline MMP-14 & $929.0 \pm 37.0$ & $162.0 \pm 2.0$ & $<0.01$ & 5.735 & $372.0 \pm 15.0$ & $81.0 \pm 7.0$ & $<0.01$ & 4.593 \\
\hline TGF- $\beta 1$ & $1,205.5 \pm 111.5$ & $212.0 \pm 22.0$ & $<0.01$ & 5.686 & $688.5 \pm 39.5$ & $115.5 \pm 4.5$ & $<0.01$ & 5.961 \\
\hline IL-5 & $1,112.0 \pm 44.0$ & $202.0 \pm 4.0$ & $<0.01$ & 5.505 & $1,073.5 \pm 90.5$ & $118.0 \pm 4.0$ & $<0.01$ & 9.097 \\
\hline IL-12 p40/p70 & $1,288.0 \pm 44.0$ & $235.5 \pm 3.5$ & $<0.01$ & 5.469 & $1,111.5 \pm 80.5$ & $118.5 \pm 12.5$ & $<0.01$ & 9.380 \\
\hline HVEM & $1,193.5 \pm 118.5$ & $219.5 \pm 1.5$ & $<0.01$ & 5.437 & $499.0 \pm 212.0$ & $68.5 \pm 1.5$ & $<0.05$ & 7.285 \\
\hline IL-5 R $\alpha$ & $339.0 \pm 65.0$ & $81.5 \pm 0.5$ & $<0.01$ & 4.160 & $370.0 \pm 37.0$ & $50.0 \pm 3.0$ & $<0.01$ & 7.400 \\
\hline RELM $\beta$ & $139.0 \pm 3.0$ & $41.5 \pm 0.5$ & $<0.01$ & 3.349 & $562.0 \pm 28.0$ & $75.0 \pm 1.0$ & $<0.01$ & 7.493 \\
\hline IL-1 R9 & $48.5 \pm 49.6$ & $465.0 \pm 5.0$ & $<0.01$ & 0.104 & $1.0 \pm 0.0$ & $29.0 \pm 3.0$ & $<0.01$ & 0.034 \\
\hline MMP-9 & $1.0 \pm 0.0$ & $15.0 \pm 3.0$ & $<0.01$ & 0.067 & $1.0 \pm 0.0$ & $219.5 \pm 21.5$ & $<0.01$ & 0.005 \\
\hline Decorin & $49.0 \pm 11.0$ & $913.5 \pm 76.5$ & $<0.01$ & 0.054 & $1.0 \pm 0.0$ & $180.0 \pm 6.0$ & $<0.01$ & 0.006 \\
\hline IL-2 R $\gamma$ & $1.0 \pm 0.0$ & $164.5 \pm 3.5$ & $<0.01$ & 0.006 & $1.0 \pm 0.0$ & $109.0 \pm 3.0$ & $<0.01$ & 0.009 \\
\hline
\end{tabular}

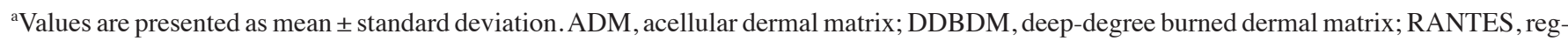
ulated on activation normal T cell and secreted; IL, interleukin; TNF- $\alpha$, tumor necrosis factor- $\alpha$; GDF-1, embryonic growth/differentiation factor 1 ; IFN- $\beta$, interferon; MCP-1, monocyte chemotactic protein-1; MMP, matrix metalloproteinase; TGF- $\beta 1$, transforming growth factor- $\beta 1$; HVEM, herpesvirus entry mediator; $\mathrm{R}$, receptor; RELM $\beta$, resistin-like molecule $\beta$.

after implantation, the ADM adhered more tightly to the subcutaneous tissue than the DDBDM, due to the formation of a fibrous coat. By the third week, the ADM and DDBDM were both covered by the dense fibrous coats and were difficult to separate from the surrounding tissues (Fig. 4B). At week four, the dense fibrous coats became thinner and the ADM and DDBDM began to be absorbed (Fig. 4B and C); the ratio of absorption area between them was not significantly different ( $\mathrm{P}=0.615$; Fig. 5).

Histological observation of the ADM and DDBDM following subcutaneous implantation. After one week of subcutaneous implantation, infiltration of inflammatory cells from the surface to the space among the collagen was observed in both tissues; however, the DDBDM exhibited more inflammatory cell infiltration than ADM near the surface. There was no obvious change in collagen distribution between the ADM and the DDBDM. The growth of fibroblasts and neovascularization were not observed in ADM or DDBDM (Fig 6A). After two weeks, the number of infiltrating inflammatory cells was markedly increased in the ADM but decreased in the DDBDM. A small number of new blood vessels were observed, which suggested neovascularization of the dermal matrix.
Meanwhile, the fibroblasts began to infiltrate and produce new collagen (Fig. 6B). In the third week, granulation tissue had formed on the ADM surface and more new blood vessels were observed. The number of fibroblasts and newly formed collagen increased while the old collagen began to degrade. In comparison, the DDBDM had thinner granulation tissue on the surface, and the process of neovascularization appeared to be slower, with a smaller number and smaller diameter of new blood vessels than in the ADM samples. Notably, numerous fibroblasts and the emergence of ordered new collagen fibers were observed in the DDBDM (Fig. 6C). Four weeks after subcutaneous implantation, more new collagen fibers emerged in place of the old ones, the majority of which had been degraded, with some forming cavities. It was observed that the DDBDM had more new collagen and less undegraded old collagen than the ADM (Fig. 6D).

\section{Discussion}

Deep-degree burn injuries can result in serious disability due to hypertrophic scar formation and skin contracture $(27,28)$. The eschar and denatured skin are typically removed following burn injury due to adverse reactions that may result in further 
Table IV. Expression values of the cytokines with significant differences between ADM and DDBDM.

\begin{tabular}{|c|c|c|c|c|}
\hline Cytokine & $\mathrm{ADM}^{\mathrm{a}}$ & DDBDM $^{\mathrm{a}}$ & Fold change & P-value \\
\hline IL-1 R9 & $465.0 \pm 5.0$ & $29.0 \pm 3.0$ & 16.034 & $<0.01$ \\
\hline Decorin & $913.5 \pm 76.5$ & $180.0 \pm 6.0$ & 5.075 & $<0.01$ \\
\hline HVEM & $219.5 \pm 1.5$ & $68.5 \pm 1.5$ & 3.204 & $<0.01$ \\
\hline CD11b & $355.5 \pm 4.5$ & $152.0 \pm 6.0$ & 2.339 & $<0.01$ \\
\hline IL-10 & $221.0 \pm 7.0$ & $98.0 \pm 1.0$ & 2.255 & $<0.01$ \\
\hline IL-12 p70 & $322.0 \pm 11.0$ & $150.0 \pm 16.0$ & 2.147 & $<0.01$ \\
\hline MCP-1 & $187.5 \pm 12.5$ & $89.5 \pm 0.5$ & 2.095 & $<0.01$ \\
\hline IL-3 & $303.0 \pm 15.0$ & $145.5 \pm 3.5$ & 2.082 & $<0.01$ \\
\hline IFN- $\gamma$ & $332.5 \pm 6.5$ & $163.0 \pm 1.0$ & 2.040 & $<0.01$ \\
\hline MMP-14 & $162.0 \pm 2.0$ & $81.0 \pm 7.0$ & 2.000 & $<0.01$ \\
\hline IL-12 p40/p70 & $235.5 \pm 3.5$ & $118.5 \pm 12.5$ & 1.987 & $<0.01$ \\
\hline TGF- $\beta 1$ & $212.0 \pm 22.0$ & $115.5 \pm 4.5$ & 1.835 & $<0.01$ \\
\hline TNF- $\alpha$ & $184.0 \pm 17.0$ & $107.0 \pm 4.0$ & 1.720 & $<0.01$ \\
\hline IL-6 & $226.0 \pm 20.0$ & $131.5 \pm 9.5$ & 1.719 & $<0.01$ \\
\hline IL-5 & $202.0 \pm 4.0$ & $118.0 \pm 4.0$ & 1.712 & $<0.01$ \\
\hline IL-17 & $180.0 \pm 8.0$ & $107.5 \pm 3.5$ & 1.674 & $<0.01$ \\
\hline IL-2 R $\gamma$ & $164.5 \pm 3.5$ & $109.0 \pm 3.0$ & 1.509 & $<0.01$ \\
\hline IL-9 & $65.5 \pm 9.5$ & $258.0 \pm 32.0$ & 0.254 & $<0.01$ \\
\hline MMP-9 & $15.0 \pm 3.0$ & $219.5 \pm 21.5$ & 0.068 & $<0.01$ \\
\hline
\end{tabular}

${ }^{a}$ Values are presented as mean \pm standard deviation. ADM, acellular dermal matrix; DDBDM, deep-degree burned dermal matrix; IL, interleukin; R, receptor; HVEM, herpesvirus entry mediator; MCP-1, monocyte chemotactic protein-1; INF- $\gamma$, interferon- $\gamma$; MMP, matrix metalloproteinase; TGF- $\beta 1$, transforming growth factor- $\beta 1$; TNF- $\alpha$, tumor necrosis factor- $\alpha$.

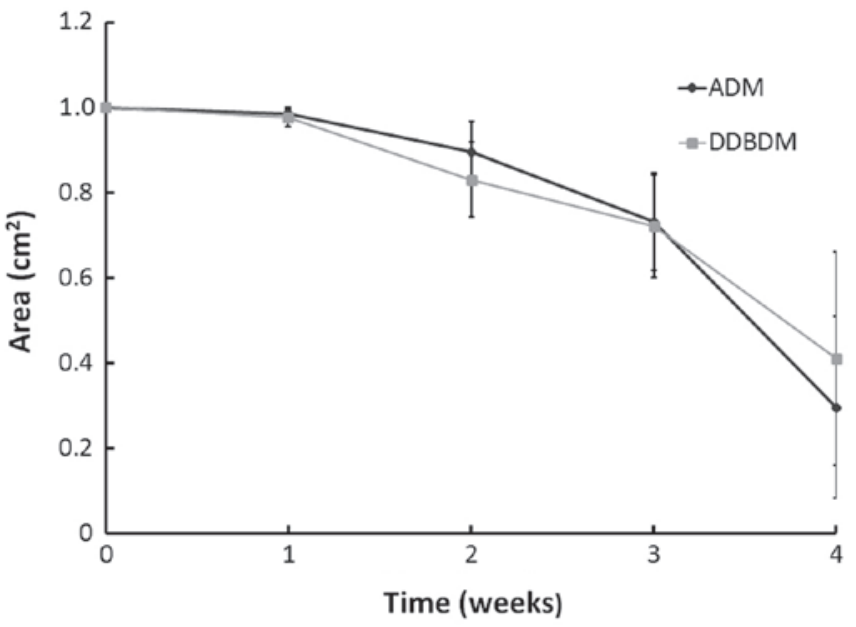

Figure 5. Area of ADM and DDBDM following subcutaneous implantation. Values are presented as mean \pm standard deviation. ADM, acellular dermal matrix; DDBDM, deep-degree burned dermal matrix.

loss of dermis. Tissue-engineered skin substitutes are generally applied to cover the burn wound following removal of the eschar, improving wound healing $(12,29,30)$. The clinical application of tissue-engineered skin substitutes or an ADM may avoid damage caused by multiple wound dressing changes and thus, accelerate wound healing and reduce scar formation $(31,32)$.

The biological mechanical properties of the dermal matrix are very important. The common treatment method of the dermal matrix is to cover the burn wound following tangential excision. As a barrier between the wound and the external environment, the dermal matrix should have adequate intensity to bear pressure and tensile. In addition, it must possess suitable elasticity such that it can return to its normal length following some degree of elongation. At present, there is no standard method for evaluating the mechanical properties of tissue-engineered skin substitutes (33-36). In the present study, there was a significant difference in the mechanical intensities of ADM and DDBDM. As compared with DDBDM, ADM exhibited enhanced tensile strength and maximum tension. Furthermore, ADM had better elasticity, which was reflected by its elongation at break and strain under stress of $3 \mathrm{MPa}$. This may have occurred due to the thermal denaturation of collagen, which may have decreased its tensile strength and extensibility. In addition, the thermal injury may have damaged the network structure of the collagen. Despite this, the gaps of these parameters between ADM and DDBDM were acceptable and it was demonstrated that DDBDM prepared in the right conditions may still possess good mechanical properties.

Tissue-engineered skin substitutes are predominantly composed of seed cells and cellular supporting structures, termed the cytoskeleton. The seed cells of the skin are fibroblasts, which produce collagen to form connective tissue, and keratinocytes, which form the epithelium. The cytoskeleton is essential to the growth of seed cells and the regeneration of tissues. It is known that the collagen is one of the most important components of the ECM. ADM is a collagen skeleton without any cells. ADM can protect burn wounds from 

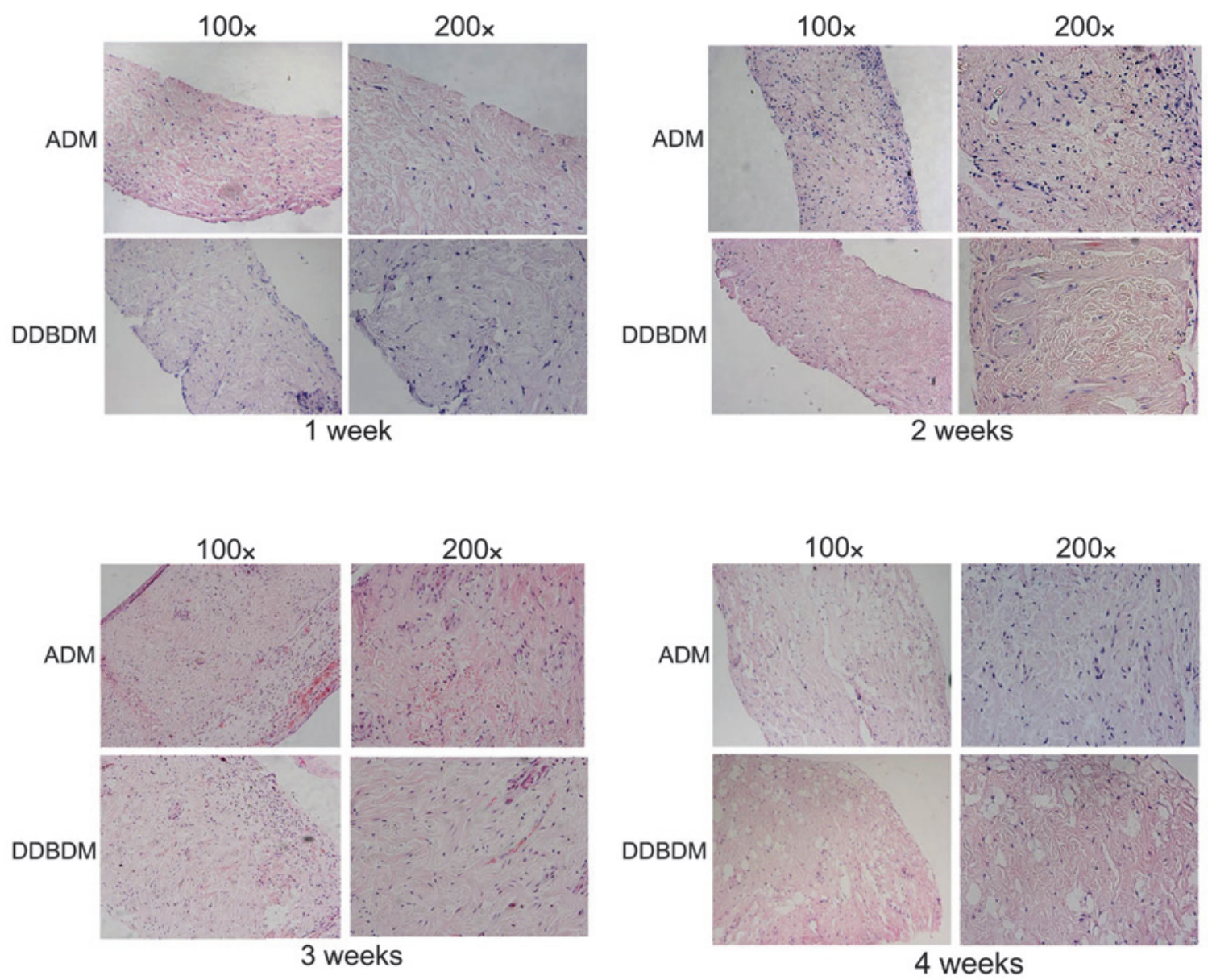

Figure 6. Histopathological observations of the ADM and DDBDM 1,2,3 and 4 weeks after subcutaneous implantation. Hematoxylin and eosin staining. ADM, acellular dermal matrix; DDBDM, deep-degree burned dermal matrix.

bacterial infection and maintain the appropriate environments to accelerate the dermis reconstruction $(7,37)$.

When the skin is deeply burned, the entire epithelium and parts of the dermis are damaged. Pathological changes to the dermis include hyaline degeneration, swelling, enlargement and disordered arrangement of collagen. When burned, the hydrogen bonds between collagen fibers break, however, the intermolecular cross-linking is not disrupted due to its low thermal sensitivity. Thus, the collagen is shortened, while the integrity of its structure is maintained $(38,39)$. When the skin is burned unevenly, there may be certain areas in which the cells are necrotic, however, the collagens are merely denatured and maintain a complete structure. Under certain conditions, these collagens may be repaired to become the ideal dermal matrix (40).

In the present study, it was observed that the collagen of DDBDM exhibited swelling and degradation, however, its structure was partially maintained. Therefore, it was hypothesized that the DDBDM may act as a satisfactory cytoskeleton with good tension and ductility when the digestion time of trypsin is sufficiently shortened. When observed microscopically, certain areas of collagen were enlarged, partially fractured and loosely arranged, but predominantly remained continuous in the DDBDM. No cells remained in the ADM and DDBDM following their removal with trypsin and Triton-X, which may explain why neither ADM nor DDBDM showed marked histoincompatibility. Almost all antigens able to induce allograft rejection were removed in the process of removing cells, and the main cells involved in allograft rejection, including T-cells and NK-cells $(41,42)$, were destroyed completely. Good histocompatibility is a critical feature of tissue-engineered tissues or organs, since they are transplanted into the human body (43-46). It has previously been demonstrated that the ECM possesses almost all the features of an ideal tissue-engineered biological material, including histocompatibility, degradability, non-toxicity and mechanical properties that match those of the original tissue $(47,48)$. In the present study, the key components of ADM or DDBDM were collagens, which are a key component of the ECM produced by fibroblasts.

A key characteristic of good histocompatibility is an inability to induce significant immune rejection responses (49-51). In the subcutaneous implantation experiment, no significant rejection response was observed for either ADM nor DDBDM, although the ADM induced a greater inflammatory reaction than DDBDM, which suggested that the so-called burn toxin did not induce adverse reactions to the DDBDM, as previously presumed. Although neovascularization was not clearly observed in the DDBDM, it exhibited a stronger ability to promote fibroblast chemotaxis and the production of new collagen. Four weeks after subcutaneous implantation with DDBDM, almost all old degenerated collagens were degraded 
and replaced by new collagen fibers with normal structure. Therefore, the DDBDM may be considered as a substitute to $\mathrm{ADM}$ to promote wound healing of patients with burns.

Burn wound healing is a complicated process involving inflammatory responses, neovascularization, granulation tissue formation, and epithelium and connective tissue remodeling $(52,53)$. When severe burns occur, a series of harmful substances, termed burn toxins are released. Burn toxins are typically products of ECM degradation that are considered to induce adverse effects in patients with burns, including methyl guanidine, histamine, putrescine, indoles and inflammatory factors. However, beneficial burn healing factors may also be produced by degradation of the ECM, possibly resulting in chemotaxis, angiogenesis, growth factor signaling and antimicrobial activities $(14,54)$. To investigate whether DDBDM, constructed from burned skin, contains reduced burn toxins and beneficial factors compared with ADM, the present study analyzed the expression of 308 cytokines in the mouse skin models.

The current study demonstrated that the expression level of certain cytokines in the skin tissue decreased significantly following burn injury, including interleukin (IL)-3, IL-10, IL-17 and decorin. By contrast, resistin-like molecule $\beta$ (RELM $\beta$ ), IL-9, IL-23 R and thrombospondin levels were increased. These cytokines are closely associated with inflammatory responses. For example, IL-10 is a known anti-inflammatory cytokine (55) and tumor necrosis factor- $\alpha$ (TNF- $\alpha$ ) is a primary mediator of the host response to inflammation (56). Decorin is expressed by fibroblasts (57) and has important biological functions, including regulating collagen formation, maintaining collagen arrangement and inhibiting the activity of transforming growth factor- $\beta$ (TGF- $\beta$ ). Reducing the level of decroin in the deep dermis may lead to hypertrophic scarring (58). A previous study demonstrated that suppressors of cytokine signaling-3 (SOCS-3) may be important in regulating the balance between immunosuppression and inflammation following thermal injury (59). IL-9 induced the expression of SOCS-3 and SOCS-3 overexpression suppressed IL-9 signaling (60). In addition, IL-9 inhibits TNF- $\alpha$ release in lipopolysaccharide-stimulated human monocytes through TGF- $\beta$ (61). TGF- $\beta$ is involved in a number of wound healing processes, including inflammation, stimulation of angiogenesis, fibroblast proliferation, collagen synthesis, and deposition and remodeling of new ECM $(62,63)$. Thrombospondin-1 and 2 are best known for their anti-angiogenic properties and their ability to modulate cell-matrix interactions (64). Thrombospondin-1 suppresses wound healing and granulation tissue formation (65), and blocks thrombospondin-1 binding to CD47, markedly increasing skin graft survival (66).

A previous study demonstrated that the expression of certain cytokines, including TNF- $\alpha$, IL-1 $\beta$ and IL-6, were significantly increased in the serum of animal models (67) and human patients with burns (68). Thus, the serum levels of TNF- $\alpha$, IL-1, IL- 6 and IL-10 may indicate the severity of this fatal condition following burn injury (69). However, in local skin tissue, the levels of these cytokines were decreased. This may be because the deep burn destroyed a large number of cells resulting in necrosis and degeneration, and the subsequent loss of cytokine expression. However, it is still unclear why RELM $\beta$, IL-9, IL-23 R and thrombospondin levels were increased in the burned skin in the current study.

To avoid the immune response induced by the genetic material of the skin donator, acellularization was performed on the normal and burned skin to decrease histoincompatibility. In the present study, the trypsin digestion and shock-elution method was successfully used to remove cells from the dermal matrix. Undergoing this process significantly decreased the levels of the majority of cytokines compared with normal and burned skin. Notably, the level of decorin was increased significantly in ADM and DDBDM compared with normal and burned skin, respectively. Decorin is a component of connective tissue that binds to type 1 collagen fibrils and is important in matrix assembly $(70,71)$. It may regulate the activity of TGF- $\beta$ as recombinant human decorin inhibits TGF- $\beta 1$-induced contraction of collagen lattices by hypertrophic scar fibroblasts (70).

In comparison, the levels of cytokines in DDBDM were lower than those in ADM, with the exception of IL-9 and MMP-9, which may explain the observation that the DDBDM induced a smaller inflammatory response than ADM in the subcutaneous implantation experiments. MMP-9 is secreted by keratinocytes and inflammatory cells, and is associated with epithelialization. A previous study also observed an increased level of MMP-9 during the early inflammatory phase of wound healing (72).

During the formation of new granulation tissue, TGF- $\beta 1$ stimulation induces the deposition of fibronectin extra domain $\mathrm{A}$ and $\alpha$-smooth muscle actin expression by fibroblasts, resulting in enhanced synthesis of new ECM (73). TGF- $\beta 1$ is also a crucial factor in the regulation of myofibroblast differentiation, facilitating the contraction of granulation tissue (74). TGF- $\beta$ has different temporal effects on wound healing and scarring, and any disruption in this expression pattern may result in hypertrophic scar formation (75). In the present study, the level of TGF- $\beta 1$ in DDBDM was lower than in ADM, which may explain the thinner granulation tissue on the surface of DDBDM compared with ADM in the second week. Excess TGF- $\beta 1$ may be responsible for the overproduction of ECM, leading to tissue fibrosis and scar formation. Therefore, the DDBDM may decrease the formation of scar tissue compared with ADM in the treatment of burns.

In conclusion, the current study describes the preparation method of DDBDM. The differences in cytokine expression between the ADM and DDBDM indicated that DDBDM did not produce increased levels of harmful burn toxin. Therefore, the DDBDM may be useful as a dermal matrix. If this method is confirmed to decrease harmful inflammatory factors and utilize beneficial factors, it may be used to alleviate adverse responses and promote wound healing processes, including seed cell infiltration, angiogenesis and matrix remodeling. The DDBDM is derived from autologous dermal matrix, therefore, the histoincompatibility associated with xenogeneic or allogeneic dermal matrices is avoided. However, the present study has a number of limitations. For example, further analysis regarding the regulation key cytokines in DDBDM were not conducted, therefore, the effect of these cytokines on the body is unknown. Additionally, it is unclear whether burn healing would be promoted using the DDBDM as a dermal matrix instead of the ADM. Future investigation should aim to resolve these questions. 


\section{Acknowledgements}

The present study was supported by the National Natural Science Foundation of China (grant nos. 30772258, 81071560 and 81372074), the Scientific and Technological Project of Shandong Province (grant no. 2009GG10002078), and Jinan Young Star Project of Science and Technology (grant no. 2013031).

\section{References}

1. Reinke JM and Sorg H: Wound repair and regeneration. Eur Surg Res 49: 35-43, 2012.

2. Orgill DP and Ogawa R: Current methods of burn reconstruction. Plast Reconstr Surg 131: 827e-836e, 2013.

3. Kagan RJ, Peck MD, A hrenholz DH, Hickerson WL, Holmes J IV, Korentager R, Kraatz J, Pollock K and Kotoski G: Surgical management of the burn wound and use of skin substitutes: an expert panel white paper. J Burn Care Res 34: e60-e79, 2013.

4. Chiu T and Burd A: "Xenograft" dressing in the treatment of burns. Clin Dermatol 23: 419-423, 2005.

5. Chiu T, Pang P, Ying SY and Burd A: Porcine skin: Friend or foe? Burns 30: 739-741, 2004.

6. Feng X, Shen R, Tan J, Chen X, Pan Y, Ruan S, Zhang F, Lin Z, Zeng Y, Wang X, et al: The study of inhibiting systematic inflammatory response syndrome by applying xenogenic (porcine) acellular dermal matrix on second-degree burns. Burns 33: 477-479, 2007.

7. Feng X, Tan J, Pan Y, Wu Q, Ruan S, Shen R, Chen X and Du Y: Control of hypertrophic scar from inception by using xenogenic (porcine) acellular dermal matrix (ADM) to cover deep second degree burn. Burns 32: 293-298, 2006.

8. Allman AJ, McPherson TB, Badylak SF, Merrill LC, Kallakury B, Sheehan C, Raeder RH and Metzger DW: Xenogeneic extracellular matrix grafts elicit a $\mathrm{TH} 2$-restricted immune response. Transplantation 71: 1631-1640, 2001.

9. Jiang D, Chen B, Xu M, Hu D, Tang C and Zhu X: The manufacturing and clinical application of heterogenous acellular dermal matrix. Zhonghua Shao Shang Za Zhi 18: 15-18, 2002 (In Chinese).

10. Philandrianos C, Andrac-Meyer L, Mordon S, Feuerstein JM, Sabatier F, Veran J, Magalon G and Casanova D: Comparison of five dermal substitutes in full-thickness skin wound healing in a porcine model. Burns 38: 820-829, 2012.

11. Truong AT, Kowal-Vern A, Latenser BA, Wiley DE and Walter RJ: Comparison of dermal substitutes in wound healing utilizing a nude mouse model. J Burns Wounds 4: e4, 2005.

12. Chen SG, Tzeng YS and Wang CH: Treatment of severe burn with DermACELL(®), an acellular dermal matrix. Int J Burns Trauma 2: 105-109, 2012.

13. Solomon JR: Early surgical excision and grafting of burns including tangential excision. Prog Pediatr Surg 14: 133-149, 1981.

14. Sparkes BG, Monge G, Marshall SL, Peters WJ, Allgöwer M and Schoenenberger GA: Plasma levels of cutaneous burn toxin and lipid peroxides in thermal injury. Burns 16: 118-122, 1990.

15. Hiramatsu M, Izawa Y, Hagihara M, Nishigaki I and Yagi K: Serum lipid peroxide levels of patients suffering from thermal injury. Burns Incl Therm Inj 11: 111-116, 1984.

16. Schoenenberger GA: Burn toxins isolated from mouse and human skin. Their characterization and immunotherapy effects. Monogr Allergy 9: 72-139, 1975.

17. Allgöwer M, Cueni LB, Städtler K and Schoenenberger GA Burn toxin in mouse skin. J Trauma 13: 95-111, 1973.

18. Constantian MB: Association of sepsis with an immunosuppressive polypeptide in the serum of burn patients. Ann Surg 188: 209-215, 1978

19. Ozkan AN and Ninnemann JL: Circulating mediators in thermal injuries: Isolation and characterization of a burn injury-induced immunosuppressive serum component. J Burn Care Rehabil 6: $147-151,1985$.

20. Schoenenberger GA, Burkhardt F, Kalberer F, Müller W, Städtler K, Vogt P and Allgöwer M: Experimental evidence for a significant impairment of host defense for gram-negative organisms by a specific cutaneous toxin produced by severe burn injuries. Surg Gynecol Obstet 141: 555-561, 1975.
21. Kremer B, Allgöwer M, Scheidegger AM, Schmidt KH, Schölmerich J, Wüst B, Schoenenberger GA: Toxin-specific ultrastructural alterations of the mouse liver after burn injuries and the possibility of a specific antitoxic therapy. Scand J Plast Reconstr Surg 13: 217-222, 1979.

22. Schölmerich J, Kremer B, Schmidt K, Setyadharma H, Richter IE and Schoenenberger GA: Effects of peptide hormones on urea- and glycogen-synthesis of isolated hepatocytes and the influence of a toxic factor from burnt mouse and human skin. Horm Metab Res 14: 80-84, 1982.

23. Ninnemann JL and Stein MD: Suppressor cell induction by povidone-iodine: In vitro demonstration of a consequence of clinical burn treatment with betadine. J Immunol 126: 1905-1908, 1981.

24. Sparkes BG, Gyorkos JW, Gorczynski RM and Brock AJ: Comparison of endotoxins and cutaneous burn toxin as immunosuppressants. Burns 16: 123-127, 1990.

25. Sparkes BG: Mechanisms of immune failure in burn injury. Vaccine 11: 504-510, 1993.

26. Wang XC, Li C, Shan F, Wang WT, Zhu XG and Jiang DY: Experimental study on the recycling of denatured acellular dermal matrix after burn. Zhonghua Shao Shang Za Zhi 28: 201-206, 2012 (In Chinese).

27. Lu KH and Li HY: Study on the management of postburn pathological scars. Zhonghua Shao Shang Za Zhi 20: 65-66, 2004 (In Chinese).

28. Eldad A, Din A, Weinberg A, Neuman A, Lipton H, Ben-Bassat H, Chaouat $\mathrm{M}$ and Wexler MR: Cryopreserved cadaveric allografts for treatment of unexcised partial thickness flame burns: Clinical experience with 12 patients. Burns 23: 608-614, 1997.

29. Purdue GF, Hunt JL, Still JM Jr, Law EJ, Herndon DN, Goldfarb IW, Schiller WR, Hansbrough JF, Hickerson WL, Himel HN, et al: A multicenter clinical trial of a biosynthetic skin replacement, Dermagraft-TC, compared with cryopreserved human cadaver skin for temporary coverage of excised burn wounds. J Burn Care Rehabil 18: 52-57, 1997 .

30. Anderson JR, Fear MW, Phillips JK, Dawson LF, Wallace H, Wood FM and Rea SM: A preliminary investigation of the reinnervation and return of sensory function in burn patients treated with INTEGRA®. Burns 37: 1101-1108, 2011.

31. Lu SL, Liao ZJ, Xiang J, Wang ZY, Yang LY and Shi JX: Clinical observation of the effect of tangential excision within 24 postburn hours on the patients with deep partial thickness burn. Zhonghua Shao Shang Za Zhi 19: 326-328, 2003 (In Chinese).

32. Wang ZQ, Cai BR, Xiao J, Hao GH, Wu JB and Zhao XH: The clinical staging and tissue bacterial quantification in the diagnosis of burn wound sepsis. Zhonghua Shao Shang Za Zhi 19: 282-284, 2003 (In Chinese).

33. Zhang Z, Lv L, Mamat M, Chen Z, Liu L and Wang Z: Xenogenic (porcine) acellular dermal matrix is useful for the wound healing of severely damaged extremities. Exp Ther Med 7: 621-624, 2014.

34. Lavine M, Frisk M and Pennisi E: Biomaterials. Introduction. Science 338: 899, 2012.

35. Mehrali M, Shirazi FS, Mehrali M, Metselaar HS, Kadri NA and Osman NA: Dental implants from functionally graded materials. J Biomed Mater Res A 101: 3046-3057, 2013.

36. Leong KF, Chua CK, Sudarmadji N and Yeong WY: Engineering functionally graded tissue engineering scaffolds. J Mech Behav Biomed Mater 1: 140-152, 2008.

37. Chen X, Shi Y, Shu B, Xie X, Yang R, Zhang L, Ruan S, Lin Y, Lin Z, Shen R, et al: The effect of porcine ADM to improve the burn wound healing. Int J Clin Exp Pathol 6: 2280-2291, 2013.

38. Pierce MC, Sheridan RL, Hyle Park B, Cense B and de Boer JF: Collagen denaturation can be quantified in burned human skin using polarization-sensitive optical coherence tomography. Burns 30: 511-517, 2004.

39. Cuttle L, Kempf M, Phillips GE, Mill J, Hayes MT, Fraser JF, Wang XQ, Kimble RM: A porcine deep dermal partial thickness burn model with hypertrophic scarring. Burns 32: 806-820, 2006.

40. Hayashi K and Markel MD: Thermal capsulorrhaphy treatment of shoulder instability: Basic science. Clin Orthop Relat Res 390: 59-72, 2001.

41. Hall BM: Cells mediating allograft rejection. Transplantation 51: 1141-1151, 1991.

42. Alegre ML, Florquin S and Goldman M: Cellular mechanisms underlying acute graft rejection: Time for reassessment. Curr Opin Immunol 19: 563-568, 2007.

43. van Minnen B, van Leeuwen MB, Stegenga B, Zuidema J, Hissink CE, van Kooten TG and Bos RR: Short-term in vitro and in vivo biocompatibility of a biodegradable polyurethane foam based on 1,4-butanediisocyanate. J Mater Sci Mater Med 16: 221-227, 2005. 
44. Pomahac B, Svensjö T, Yao F, Brown H and Eriksson E: Tissue engineering of skin. Crit Rev Oral Biol Med 9: 333-344, 1998.

45. Prasad T, Shabeena EA, Vinod D, Kumary TV and Anil Kumar PR: Characterization and in vitro evaluation of electrospun chitosan/polycaprolactone blend fibrous mat for skin tissue engineering. J Mater Sci Mater Med 26: 5352, 2015.

46. Mañez R, White LT, Linden P, Kusne S, Martin M, Kramer D, Demetris AJ, Van Thiel DH, Starzl TE and Duquesnoy RJ: The influence of HLA matching on cytomegalovirus hepatitis and chronic rejection after liver transplantation. Transplantation 55: 1067-1071, 1993.

47. Borschel GH, Dennis RG and Kuzon WM Jr: Contractile skeletal muscle tissue-engineered on an acellular scaffold. Plast Reconstr Surg 113: 595-602; discussion 603-604, 2004.

48. Badylak SF: The extracellular matrix as a biologic scaffold material. Biomaterials 28: 3587-3593, 2007.

49. Low PS, Tjin MS and Fong E: Design and Construction of Artificial Extracellular Matrix (aECM) Proteins from Escherichia coli for Skin Tissue Engineering. J Vis Exp: e52845, 2015.

50. Sundaramurthi D, Krishnan UM and Sethuraman $S$ : Biocompatibility of poly(3-hydroxybutyrate-co-3-hydroxyvalerate) (PHBV) nanofibers for skin tissue engineering. J Biomed Nanotechnol 9: 1383-1392,2013.

51. Müller WE and Müller IM: Origin of the metazoan immune system: Identification of the molecules and their functions in sponges. Integr Comp Biol 43: 281-292, 2003.

52. Singer AJ and Clark RA: Cutaneous wound healing. N Engl J Med 341: 738-746, 1999

53. Velnar T, Bailey T and Smrkolj V: The wound healing process: An overview of the cellular and molecular mechanisms. J Int Med Res 37: 1528-1542, 2009.

54. Chang KC, Ma H, Liao WC, Lee CK, Lin CY and Chen CC: The optimal time for early burn wound excision to reduce pro-inflammatory cytokine production in a murine burn injury model. Burns 36: 1059-1066, 2010

55. Drost AC, Burleson DG, Cioffi WG Jr, Mason AD Jr and Pruitt BA Jr: Plasma cytokines after thermal injury and their relationship to infection. Ann Surg 218: 74-78, 1993.

56. Beutler B and Cerami A: The biology of cachectin/TNF--a primary mediator of the host response. Annu Rev Immunol 7: 625-655, 1989.

57. Tan EM, Hoffren J, Rouda S, Greenbaum S, Fox JW IV, Moore JH Jr and Dodge GR: Decorin, versican, and biglycan gene expression by keloid and normal dermal fibroblasts: differential regulation by basic fibroblast growth factor. Exp Cell Res 209: 200-207, 1993.

58. Honardoust D, Varkey M, Marcoux Y, Shankowsky HA and Tredget EE: Reduced decorin, fibromodulin, and transforming growth factor- $\beta 3$ in deep dermis leads to hypertrophic scarring. J Burn Care Res 33: 218-227, 2012.

59. Ogle CK, Kong F, Guo X, Wells DA, Aosasa S, Noel G and Horseman N: The effect of burn injury on suppressors of cytokine signalling. Shock 14: 392-399, 2000.

60. Lejeune D, Demoulin JB and Renauld JC: Interleukin 9 induces expression of three cytokine signal inhibitors: Cytokine-inducible SH2-containing protein, suppressor of cytokine signalling (SOCS) -2 and SOCS-3, but only SOCS-3 overexpression suppresses interleukin 9 signalling. Biochem J 353: 109-116, 2001.

61. Pilette C, Ouadrhiri Y, Van Snick J, Renauld JC, Staquet P, Vaerman JP and Sibille Y: IL-9 inhibits oxidative burst and TNF-alpha release in lipopolysaccharide-stimulated human monocytes through TGF-beta. J Immunol 168: 4103-4111, 2002.
62. Nall AV, Brownlee RE, Colvin CP, Schultz G, Fein D, Cassisi NJ, Nguyen $\mathrm{T}$ and Kalra A: Transforming growth factor beta 1 improves wound healing and random flap survival in normal and irradiated rats. Arch Otolaryngol Head Neck Surg 122: 171-177, 1996.

63. Roberts AB, Sporn MB, Assoian RK, Smith JM, Roche NS, Wakefield LM, Heine UI, Liotta LA, Falanga V and Kehrl JH: Transforming growth factor type beta: rapid induction of fibrosis and angiogenesis in vivo and stimulation of collagen formation in vitro. Proc Natl Acad Sci USA 83: 4167-4171, 1986.

64. Bornstein P, Agah A and Kyriakides TR: The role of thrombospondins 1 and 2 in the regulation of cell-matrix interactions, collagen fibril formation, and the response to injury. Int $\mathrm{J}$ Biochem Cell Biol 36: 1115-1125, 2004.

65. Streit M, Velasco P, Riccardi L, Spencer L, Brown LF, Janes L, Lange-Asschenfeldt B, Yano K, Hawighorst T, Iruela-Arispe L, Detmar M: Thrombospondin-1 suppresses wound healing and granulation tissue formation in the skin of transgenic mice. EMBO J 19: 3272-3282, 2000

66. Isenberg JS, Pappan LK, Romeo MJ, Abu-Asab M, Tsokos M, Wink DA, Frazier WA and Roberts DD: Blockade of thrombospondin-1-CD47 interactions prevents necrosis of full thickness skin grafts. Ann Surg 247: 180-190, 2008.

67. Emanuele NV, LaPaglia N, Kovacs EJ and Emanuele MA: The impact of burn injury and ethanol on the cytokine network of the mouse hypothalamus: Reproductive implications. Cytokine 30: 109-115, 2005.

68. de Bandt JP, Chollet-Martin S, Hernvann A, Lioret N, du Roure LD, Lim SK, Vaubourdolle M, Guechot J, Saizy R, Giboudeau J and Cynober L: Cytokine response to burn injury: Relationship with protein metabolism. J Trauma 36: 624-628, 1994.

69. Drost AC, Burleson DG, Cioffi WG Jr, Jordan BS, Mason AD Jr and Pruitt BA Jr: Plasma cytokines following thermal injury and their relationship with patient mortality, burn size, and time postburn. J Trauma 35: 335-339, 1993.

70. Zhang Z, Garron TM, Li XJ, Liu Y, Zhang X, Li YY and Xu WS: Recombinant human decorin inhibits TGF-betal-induced contraction of collagen lattice by hypertrophic scar fibroblasts. Burns 35: 527-537, 2009.

71. Chen S, Young MF, Chakravarti S and Birk DE: Interclass small leucine-rich repeat proteoglycan interactions regulate collagen fibrillogenesis and corneal stromal assembly. Matrix Biol 35: 103-111, 2014.

72. Gillard JA, Reed MW, Buttle D, Cross SS and Brown NJ: Matrix metalloproteinase activity and immunohistochemical profile of matrix metalloproteinase- 2 and -9 and tissue inhibitor of metalloproteinase-1 during human dermal wound healing. Wound Repair Regen 12: 295-304, 2004.

73. Serini G, Bochaton-Piallat ML, Ropraz P, Geinoz A, Borsi L, Zardi L and Gabbiani G: The fibronectin domain ED-A is crucial for myofibroblastic phenotype induction by transforming growth factor-beta1. J Cell Biol 142: 873-881, 1998

74. Smith PC, Cáceres M and Martinez J: Induction of the myofibroblastic phenotype in human gingival fibroblasts by transforming growth factor-beta1: Role of RhoA-ROCK and c-Jun N-terminal kinase signaling pathways. J Periodontal Res 41: 418-425, 2006.

75. Lu L, Saulis AS, Liu WR, Roy NK, Chao JD, Ledbetter S and Mustoe TA: The temporal effects of anti-TGF-beta1, 2, and 3 monoclonal antibody on wound healing and hypertrophic scar formation. J Am Coll Surg 201: 391-397, 2005. 